\title{
Permeation of the three aromatic dipeptides through lipid bilayers: Experimental and computational study
}

Brent L. Lee, Krzysztof Kuczera, C. Russell Middaugh, and Gouri S. Jas

Citation: The Journal of Chemical Physics 144, 245103 (2016);

View online: https://doi.org/10.1063/1.4954241

View Table of Contents: http://aip.scitation.org/toc/jcp/144/24

Published by the American Institute of Physics

\section{Articles you may be interested in}

Publisher's Note: "Permeation of the three aromatic dipeptides through lipid bilayers: Experimental and computational study" [J. Chem. Phys. 144, 245103 (2016)]

The Journal of Chemical Physics 145, 059902 (2016); 10.1063/1.4960183

Accelerating potential of mean force calculations for lipid membrane permeation: System size, reaction coordinate, solute-solute distance, and cutoffs

The Journal of Chemical Physics 145, 125101 (2016); 10.1063/1.4963192

Calculating the free energy of transfer of small solutes into a model lipid membrane: Comparison between metadynamics and umbrella sampling

The Journal of Chemical Physics 143, 144108 (2015); 10.1063/1.4932159

A molecular-dynamics study of lipid bilayers: Effects of the hydrocarbon chain length on permeability

The Journal of Chemical Physics 123, 184714 (2005); 10.1063/1.2102900

Electron transfer reaction dynamics in non-Debye solvents

The Journal of Chemical Physics 109, 2325 (1998); 10.1063/1.476800

One-dimensional potential of mean force underestimates activation barrier for transport across flexible lipid membranes

The Journal of Chemical Physics 139, 134906 (2013); 10.1063/1.4823500

\section{AIP $\mid$ The Journal of Chemical Physics}




\title{
Permeation of the three aromatic dipeptides through lipid bilayers: Experimental and computational study
}

\author{
Brent L. Lee, ${ }^{1}$ Krzysztof Kuczera, ${ }^{1,2}$ C. Russell Middaugh, ${ }^{3}$ and Gouri S. Jas ${ }^{3, a)}$ \\ ${ }^{1}$ Department of Chemistry, The University of Kansas, Lawrence, Kansas 66045, USA \\ ${ }^{2}$ Department of Molecular Biosciences, The University of Kansas, Lawrence, Kansas 66045, USA \\ ${ }^{3}$ Department of Pharmaceutical Chemistry, The University of Kansas, Lawrence, Kansas 66047, USA
}

(Received 4 April 2016; accepted 7 June 2016; published online 27 June 2016; publisher error corrected 28 June 2016)

\begin{abstract}
The time-resolved parallel artificial membrane permeability assay with fluorescence detection and comprehensive computer simulations are used to study the passive permeation of three aromatic dipeptides $-N$-acetyl-phenylalanineamide (NAFA), $N$-acetyltyrosineamide (NAYA), and $N$-acetyltryptophanamide (NATA) through a 1,2-dioleoyl-sn-glycero-3-phospocholine (DOPC) lipid bilayer. Measured permeation times and permeability coefficients show fastest translocation for NAFA, slowest for NAYA, and intermediate for NATA under physiological temperature and $\mathrm{pH}$. Computationally, we perform umbrella sampling simulations to model the structure, dynamics, and interactions of the peptides as a function of $\mathrm{z}$, the distance from lipid bilayer. The calculated profiles of the potential of mean force show two strong effects-preferential binding of each of the three peptides to the lipid interface and large free energy barriers in the membrane center. We use several approaches to calculate the position-dependent translational diffusion coefficients $\mathrm{D}(\mathrm{z})$, including one based on numerical solution the Smoluchowski equation. Surprisingly, computed $D(z)$ values change very little with reaction coordinate and are also quite similar for the three peptides studied. In contrast, calculated values of sidechain rotational correlation times $\tau_{\text {rot }}(\mathrm{z})$ show extremely large changes with peptide membrane insertion - values become 100 times larger in the headgroup region and 10 times larger at interface and in membrane center, relative to solution. The peptides' conformational freedom becomes systematically more restricted as they enter the membrane, sampling $\alpha$ and $\beta$ and $\mathrm{C}_{7 \text { eq }}$ basins in solution, $\alpha$ and $\mathrm{C}_{7 \mathrm{eq}}$ at the interface, and $\mathrm{C}_{7 \mathrm{eq}}$ only in the center. Residual waters of solvation remain around the peptides even in the membrane center. Overall, our study provides an improved microscopic understanding of passive peptide permeation through membranes, especially on the sensitivity of rotational diffusion to position relative to the bilayer. Published by AIP Publishing. [http://dx.doi.org/10.1063/1.4954241]
\end{abstract}

\section{INTRODUCTION}

Biological membranes form the basis of all multicellular life. They regulate the intracellular and extracellular environment by serving as the gatekeepers for the passage of molecules through passive diffusion, facilitated diffusion, and active transport. Modern evolutionary theory predicts that the first cellular transport machinery developed from the passive diffusion of proteins across a simple lipid bilayer. ${ }^{1,2}$ Roughly $30 \%$ of all proteins encoded by the human genome are membrane proteins ${ }^{3}$ and $70 \%$ of all modern pharmaceutical targets aim to influence and regulate these processes. ${ }^{4}$ Even drugs that do not interact with cell membranes must pass through this barrier in order to reach their intracellular targets. ${ }^{5,6}$ As a result, a fundamental knowledge of the passive diffusion of small peptides is essential towards understanding all of these processes on both a theoretical and practical level. Rather than taking a macromolecular approach, we focus on the basic building blocks of these larger constructs-amino acids.

\footnotetext{
a) Author to whom correspondence should be addressed. Electronic mail: 112gjas@uccaribe.edu
}

Unfortunately, little is known about the passive diffusion of many amino acids, which serve as the building blocks for proteins. Experimental methods using planar bilayer and liposome systems with detection through a wide range of approaches have been used extensively to study the interfacial regions of membranes ${ }^{7-9}$ but tend to lack atomistic detail of the processes involved within the membrane. The Parallel Artificial Membrane Permeation Assay (PAMPA) method uses polycarbonate microporous support with a single lipid bilayer per pore. ${ }^{10-13}$ The permeation rates of samples also correspond to the Caco- 2 model in intestinal absorption and clinical studies of the blood-brain barrier. The components of the lipid bilayer can also be deduced and separated. This enables the measurement of translocation of samples through the corresponding lipid bilayer. The interaction between the permeant and the bilayer lipids and the mechanism of the membrane translocation may be elucidated through this filtersupported bilayer lipid system.

As computational power and empirical force field parameterization have improved, atomistic level molecular dynamics (MD) simulations have gained greater prominence towards studying the diffusion of small molecules through a membrane. ${ }^{14}$ Recent molecular dynamics studies have 
focused on a wide variety of molecules passively diffusing through membranes, such as water, ${ }^{15-17}$ small molecules, ${ }^{18-23}$ model drug compounds, ${ }^{6,22,24,25}$ analgesics, ${ }^{26-28}$ drug delivery systems, ${ }^{29,30}$ dyes, ${ }^{31,32}$ other lipids, ${ }^{33,34}$ nanoparticles, ${ }^{35-37}$ toxins, ${ }^{38}$ small peptides, ${ }^{39,40}$ and even transmembrane proteins. ${ }^{41,42}$ However, only a handful of MD studies have examined amino acid-related systems and are confined to tryptophan, ${ }^{43-45}$ arginine,${ }^{46,47}$ lysine, ${ }^{47}$ and amino acid analogues. ${ }^{48}$ In terms of potential of mean force (PMF), findings have consistently shown that small nonpolar molecules tend to be preferentially bound in the membrane center, while polar molecules tend to interact favorably with the lipid headgroups and experience a free energy barrier in the center. The PMFs for the sidechains of tryptophan, tyrosine, and phenylalanine determined by MacCallum et al. mostly fit this general picture, with tryptophan and tyrosine exhibiting PMF minima at the water-lipid interface. Both phenylalanine and tyrosine have lower free energies inside the lipid than in water, while tryptophan has to overcome a significant free energy barrier in the membrane center. In studies of blocked tryptophan $(\mathrm{N}$ acetyltryptophanamide or NATA), ${ }^{43}$ a similar behavior was found as for the tryptophan sidechain, with NATA exhibiting a significantly higher barrier in the central region.

The goal of our study is to expand the understanding of permeation of small amino acids through lipid membranes. We present results of experiments and computer simulations for blocked forms of the three aromatic dipeptides-NATA, $N$-acetyltyrosineamide (NAYA), and $\mathrm{N}$-acetylphenylalanineamide (NAFA) in 1,2-dioleoyl-snglycero-3-phospocholine (DOPC) lipid bilayers (Fig. 1). Experimentally, we measure the permeability coefficients and permeation times of NAFA, NAYA, and NATA through a DOPC bilayer using the PAMPA method on a polyvinylidene fluoride (PVDF) support. ${ }^{10-13}$ In the computational part, we employ molecular dynamics simulations with umbrella sampling to explore the structures, motions, and interactions of the systems as a function of distance from membrane center, including new approaches to calculating the positiondependent diffusion coefficients $\mathrm{D}(\mathrm{z})$ and analysis of rotational diffusion. The amphiphilic dipeptides exhibit interesting and unexpected properties. The PMFs for NATA, NAYA, and NAFA follow the form previously found for polar molecules, with a minimum at the interface and barrier in the center. Surprisingly, the rate of translational diffusion $\mathrm{D}(\mathrm{z})$ is mostly flat, exhibiting only small changes with depth of membrane insertion z. However, the reorientation rates of the dipeptides do exhibit significant changes with $z$, becoming markedly slower inside the bilayer and dramatically slower at the waterlipid interface, compared to the solvent phase. Additionally, as peptides move from the solvent to the interface, headgroup, and tail regions, they undergo characteristic conformational changes in response to the changing environment. Overall, our joint experimental and computational study provides a new level of understanding of the mechanism of passive permeation of aromatic peptides through lipid bilayers.

\section{METHODS}

\section{Materials}

1,2-Dioleoyl-sn-glycero-3-phosphocholine (DOPC) was purchased from Avanti Polar Lipids (Alabaster, AL). The

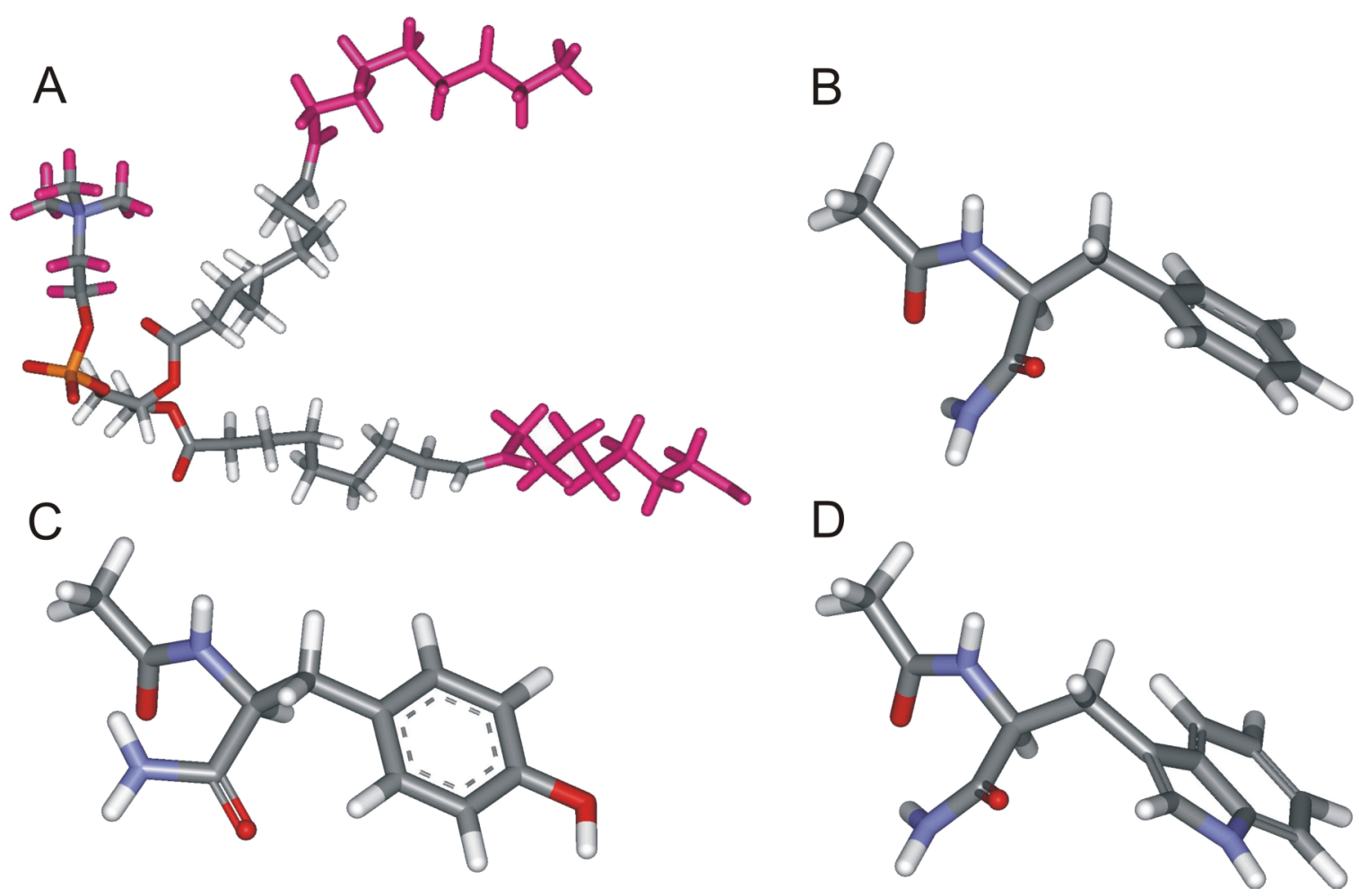

FIG. 1. Chemical structures of studied systems. (a) 1,2-dioleoyl-sn-glycero-3-phospocholine (DOPC), (b) $N$-acetylphenylalanineamide (Ac-Phe-NH 2 or NAFA), (c) $N$-acetyltyrosineamide (Ac-Tyr- $\mathrm{NH}_{2}$ or NAYA), (d) $N$-acetyltryptophanamide (Ac-Trp- $\mathrm{NH}_{2}$ or NATA). 
NAFA, NATA, NAYA, sodium acetate, sodium phosphate monobasic, sodium phosphate dibasic, phosphate buffered saline tablets (P-4417), 1,9-decadiene (Aldrich 118303), cresyl blue, and lucifer yellow were purchased from Sigma (St. Louis, MO). Hydrophobic filter plates $(0.45 \mu \mathrm{m}$ PVDF Membrane without underdrain; MAIPNTR10) and the 96well disposable transport receiver plates (MATRNPS50) were purchased from Millipore Corporation (Billerica, MA).

\section{Experimental}

The parallel artificial permeation assay (PAMPA) was used to study permeation of bio-molecules through lipid bilayer, originally proposed by Kansy et al. ${ }^{10}$ This procedure has provided a straightforward approach to measure unassisted permeation and has been widely used to study oral absorption, blood-brain barrier crossing, and permeation through skin. The PAMPA method was employed, as described previously. ${ }^{10,49-54}$ Peptides were prepared at a concentration of $180 \mu \mathrm{M}$ for NATA and $\sim 600 \mu \mathrm{M}$ for NAYA and NAFA. All samples were prepared in both $20 \mathrm{mM}$ sodium acetate buffer at $\mathrm{pH} 4.8$ and $20 \mathrm{mM}$ sodium phosphate buffer at $\mathrm{pH}$ 7.2. Lipid solutions $(5 \% \mathrm{w} / \mathrm{v})$ of DOPC were prepared in 1,9-decadiene. Sonication was applied to DOPC to ensure complete solvation. A 96-well acceptor microplate was filled with $280 \mu \mathrm{l}$ of the corresponding buffer, and the PVDF filter plate (donor compartment) was fused on the buffer-filled acceptor plate. The entire experiment was carried out at $309 \mathrm{~K}$. The lipid solution $(5 \mu \mathrm{l})$ was carefully added onto the filter surface and, immediately after, the corresponding peptide solutions ( $150 \mu \mathrm{l}$ per each well) were placed on the filter plate. Samples and lipid were pipetted every $3 \mathrm{~h}$ and the experiment was terminated at $30 \mathrm{~h}$. The fluorescence intensity of the permeated sample was measured with a Fluorolog (Horiba Jobin Yvon, Inc., Edison, $\mathrm{NJ}$ ) with the excitation wavelength set to the corresponding absorption maxima $(\mathrm{NAFA}=257 \mathrm{~nm}$, NATA $=280 \mathrm{~nm}$, NAYA $=276 \mathrm{~nm}$ ). Membrane integrity was tested with Lucifer yellow and Cresyl blue as described previously. Experimental $\mathrm{P}$ values were determined spectroscopically by measuring sample concentrations before and after migration with a specific delay time through the bi-layer surface area $\left(0.28 \mathrm{~cm}^{2}\right)$. All sample concentrations were determined by the measured absorbance and molar extinction coefficients of the corresponding samples at a specific wavelength [NAFA $(257 \mathrm{~nm})$, NATA $(280 \mathrm{~nm})$, and NAYA $(276 \mathrm{~nm})]$.

\section{Computational}

The simulated peptides were $N$-acetyltryptophanamide (Ac-Trp- $\mathrm{NH}_{2}$ or NATA), $N$-acetyltyrosineamide (Ac-Tyr- $\mathrm{NH}_{2}$ or NAYA), and $N$-acetylphenylalanineamide (Ac-Phe- $\mathrm{NH}_{2}$ or NAFA) (Fig. 1). Initial peptide structures were built with CHARMM $^{55,56}$ in extended conformations. The phospholipid bilayers with a single copy of peptide in aqueous phase were created by using CHARMM-GUI ${ }^{57-59}$ and all molecular dynamics simulations were conducted with GROMACS 4.5.4 or 4.5.6. ${ }^{60}$ The bilayer systems contained the following molecules: $50(2 \times 25)$ 1,2-dioleoyl-sn-glycero-3-phosphocholine
(DOPC) molecules, one peptide, eight chloride, and eight sodium ions and TIP3P water. The sodium and chloride ions were added to maintain a physiologically relevant ionic strength. Due to small variations in system size, the phenylalanine, tyrosine, and tryptophan simulations contained 2939, 2949, and 2599 TIP3P water molecules, respectively, in tetragonal boxes with dimensions of $4.29 \times 4.29$ $\times 8.30 \mathrm{~nm}, 4.22 \times 4.22 \times 8.56 \mathrm{~nm}$, and $4.19 \times 4.19 \times 8.09$ $\mathrm{nm}$, respectively. These boxes produce DOPC headgroup areas of $0.7355 \mathrm{~nm}^{2}, 0.7122 \mathrm{~nm}^{2}$, and $0.7017 \mathrm{~nm}^{2}$, respectively, in good agreement with experimental averages of $0.723 \mathrm{~nm}^{2} .{ }^{61,62}$ The electron density profile of these membranes is also in agreement with experimental profiles (see supplementary material). ${ }^{62}$ In our coordinate system, $\mathrm{x}$ and $\mathrm{y}$ axes are in the membrane plane and $\mathrm{z}$ is the plane normal. DOPC and peptide molecular interactions were represented by the CHARMM v.36 force field ${ }^{63,64}$ and water was described by the TIP3P model. ${ }^{65}$

Phenylalanine and tyrosine simulations were performed using GROMACS 4.5.4, and all tryptophan simulations were performed with GROMACS 4.5.6. ${ }^{60}$ Periodic boundary conditions were used along all three coordinate axes. Direct electrostatic interactions were cut off at $0.13 \mathrm{~nm}$, with long range effects calculated by using the particle mesh Ewald method with a mesh spacing of $0.12 \mathrm{~nm}$. van der Waals interactions were truncated at $1.2 \mathrm{~nm}$, smoothed with a switching function between 1.0 and $1.2 \mathrm{~nm}$. Newton's equations of motion were integrated by using the default leap-frog algorithm with a time step of $2 \mathrm{fs}$ and constraints on all bonds using the LINCS algorithm. ${ }^{66}$ Temperature control was achieved by using velocity rescaling. ${ }^{67}$ The position of the permeant was recorded every $0.1 \mathrm{ps}$, the permeant pulling force every $0.2 \mathrm{ps}$, and complete structural information for the entire system every $1.0 \mathrm{ps}$. Initial velocities were determined from a Maxwell distribution at $300 \mathrm{~K}$. Temperatures were kept constant by using velocity rescaling with an added stochastic term. ${ }^{67}$ All simulations were run with an NVT ensemble. The initial simulation image was equilibrated over $500 \mathrm{ps}$ intervals with increasingly more stringent restraints. An unrestrained molecular dynamics simulation was then run for $120 \mathrm{~ns}$. A system image was then extracted where the peptide permeant was located $1.6 \mathrm{~nm}$ from the lipid bilayer center and was then used as the starting point for successive umbrella sampling windows.

Successive umbrella sampling windows were conducted at distances from 0 to $3.0 \mathrm{~nm}$ away from center of the lipid bilayer and along the $\mathrm{z}$-axis. The phenylalanine and tryptophan windows were spaced in even $0.1 \mathrm{~nm}$ increments for a total of 31 different positions. Windows for tyrosine were run at $0.0 \mathrm{~nm}$; from 0.09 to $0.9 \mathrm{~nm}$ in $0.09 \mathrm{~nm}$ increments; and then from 1.0 to $3.0 \mathrm{~nm}$ in $0.1 \mathrm{~nm}$ increments. The tighter increment spacing was used to improve statistical sampling and overlap between simulation windows. For phenylalanine and tryptophan, this was accomplished by gradually increasing the simulation length from $50 \mathrm{~ns}$ to $100 \mathrm{~ns}$ as the peptide neared the center of the lipid bilayer. All simulation windows were run for at least $50 \mathrm{~ns}$. A restraining umbrella sampling potential of $3000 \mathrm{~kJ} \mathrm{~mol}^{-1} \mathrm{~nm}^{-1}$ was applied to peptide center of mass, with minimum at the center of each window. ${ }^{68,69}$ 
The weighted histogram analysis method (WHAM) was then applied to the resulting data to obtain the potential of mean force (PMF). ${ }^{70-72}$

Position dependent translation diffusion coefficients $\mathrm{D}(\mathrm{z})$ were determined using three approaches-one based on the Smoluchowski equation and two on the fluctuation-dissipation theorem. The first approach uses a numerical solution to the Smoluchowski equation as described by Bicout and Szabo ${ }^{73}$ and as discussed by Hummer. ${ }^{74}$ The values of the centerof-mass distance between peptide and membrane $\mathrm{z}(\mathrm{t})$ within each umbrella sampling window are histogrammed, yielding the biased probability distribution $\mathrm{p}^{*}(\mathrm{z})=\mathrm{p}^{*}(\mathrm{n})$, where $\mathrm{n}$ is the bin number. The transition rates between neighboring bins, $\mathrm{w}_{\mathrm{n}+1, \mathrm{n}}$ are calculated from the number of transitions in $\mathrm{z}(\mathrm{t})$ and the bin residence times. Diffusion coefficients are then calculated as

$$
D_{n+1 / 2}=d^{2} w_{n+1, n}\left(\frac{p^{*}(n)}{p^{*}(n+1)}\right)^{1 / 2},
$$

with $\mathrm{d}$ being the bin width $(\mathrm{d}=0.02 \mathrm{~nm}$ was used). These coefficients correspond to motion on the biased potential, including the harmonic US restraint. However, from each simulation, we only take the $\mathrm{D}(\mathrm{z})$ value at the window center, where the constraint potential is approximately 0. Errors were estimated by performing separate calculations over four quarters of the data and by multiplying the standard error of the mean by the appropriate t-coefficient at the $95 \%$ confidence level (3.182).

The two other methods of calculating $\mathrm{D}(\mathrm{z})$ were based on the fluctuation-dissipation theorem, ${ }^{75}$

$$
D(z)=\frac{\left(k_{B} T\right)^{2}}{\int_{0}^{\infty}\left\langle\Delta F_{z}(z, 0) \Delta F_{z}(z, t)\right\rangle d t},
$$

where $\mathrm{k}_{\mathrm{B}}$ is the Boltzmann constant, $\mathrm{T}$ is the temperature, and $\Delta \mathrm{F}_{\mathrm{z}}(\mathrm{z}, \mathrm{t})$ is the deviation of the $\mathrm{z}$-component of the force experienced on the center of mass of the permeant from its average value, as a function of the permeant depth, $z$, and time, $t$. These two approaches differed in terms of the forces used. In the first, forces corresponding to fixed values of $\mathrm{z}$ were calculated, using a custom modified version of CHARMM v. 38, designed to enable fixing the difference in COM z-coordinates between two sub-systems. For each US window, ten independent MD trajectories were generated with the same CHARMM v. 36 protein and lipid parameters as used in the GROMACS simulations. ${ }^{56}$ The starting images were extracted from the umbrella sampling trajectories. The CHARMM simulations were then run for $100 \mathrm{ps}$ with a time step of 2 fs and force data were recorded every 0.1 ps. Temperature was kept constant at $300 \mathrm{~K}$ by using a Nose-Hoover thermostat. ${ }^{76,77}$ Force autocorrelation functions were then numerically integrated until temporal convergence was achieved, typically after 15 ps. This approach to $\mathrm{D}(\mathrm{z})$ estimation is analogous to that employed in the original constrained-z simulations of Marrink and Berendsen. ${ }^{15}$ The final method for obtaining $\mathrm{D}(\mathrm{z})$ used autocorrelations of the umbrella restraining force, recorded for each US window. In this case numerical integration typically converged after $1000 \mathrm{ps}$. The $\mathrm{D}(\mathrm{z})$ values from the Smoluchowski equation and the fixed-z forces method agreed throughout the simulation range, within errors. The values obtained in the aqueous phase with these methods also agreed with a separate MD simulation of NATA in a TIP3P box (see Results and Discussion). The translational diffusion coefficients obtained from fluctuations of the restraint force were typically 5-6 times lower than those of the first two methods, and did not agree with the free NATA MD in water (see supplementary material). Thus, the last method of $\mathrm{D}(\mathrm{z})$ calculation was not used in further analysis.

The inhomogeneous solubility diffusion model was then used to calculate the permeability coefficient $\mathrm{P}$ and mean first passage time (MFPT) $\langle\tau\rangle,{ }^{15,19,43,78}$

$$
P=\left[\int_{a}^{b} \frac{e^{\beta w(z)}}{D(z)} d z\right]^{-1},
$$

where $w(z)$ is the potential of mean force at location $z$, $a$ is the $z$ location of the free energy minimum along the membrane interface, $b$ is the opposite side of the membrane, and $\beta=\left(\mathrm{k}_{\mathrm{B}} \mathrm{T}\right)^{-1}$. The mean free passage time, $\langle\tau\rangle$, can then be determined as follows: $:^{79,80}$

$$
\langle\tau\rangle=\int_{a}^{b}\left[\frac{e^{\beta w(z)}}{D(z)} \int_{a}^{z} e^{-\beta w\left(z^{\prime}\right)} d z^{\prime}\right] d z .
$$

Rotational motion was studied by following reorientations of two molecular axes for each peptide. For sidechains, the axes were related to the electronic transition dipoles: the in-plane axes perpendicular to the CG-CZ vector for NAFA and NAYA, and the ${ }^{1} \mathrm{~L}_{\mathrm{b}}$ transition dipole axis for NATA. ${ }^{81}$ For the overall reorientation, the axis was the vector connecting the center of mass of the backbone to the center of mass of the sidechain. For each case, the autocorrelation function $\mathrm{C}_{2}(\mathrm{t})=1 / 2\left\langle 3 \cos ^{2}(\theta)-1\right\rangle$ was calculated, with $\theta$ being the angle of axis reorientation during time t. The rotational correlation time $\tau_{\text {rot }}$ was calculated as the integral of $\mathrm{C}_{2}(\mathrm{t})$ over a time range where the function decays to zero and the integral reaches a stable value. In the headgroup region in several cases the autocorrelation functions did not converge to zero, indicating that the sidechain reorientations were not completely sampled during the simulation period. Thus, the longest calculated correlation times are highly approximate.

\section{RESULTS AND DISCUSSION}

\section{Experimental}

The permeation of the three peptides across the DOPC bilayer was examined over a period of 30 hours. Permeation as a function of time, permeation rates, and permeability coefficients of NAFA, NATA, and NAYA is presented in

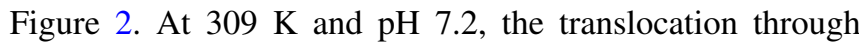
DOPC occurs with a time constant of $5 \mathrm{~h}$ for NAFA (Fig. 2(a)), $7 \mathrm{~h}$ for NATA (Fig. 2(b)), and $9.5 \mathrm{~h}$ for NAYA (Fig. 2(c)). Insets in Figures 2(a)-2(c) represent peptide migration at $\mathrm{pH}$ 4.8. At this lower $\mathrm{pH}$, translocation is slightly slower for NAFA and NAYA and slightly faster for NATA, compared to $\mathrm{pH}$ 7.2. Of the three peptides studied, NAYA exhibits the slowest DOPC permeation rate and NAFA the fastest. NAYA migrates slightly faster at $\mathrm{pH} 7.2(9.5 \mathrm{~h})$ than at a lower $\mathrm{pH}$ of $4.8(11 \mathrm{~h})$. Figure 2(d) shows the rate constants, obtained by fitting the permeation times to exponential curves, 

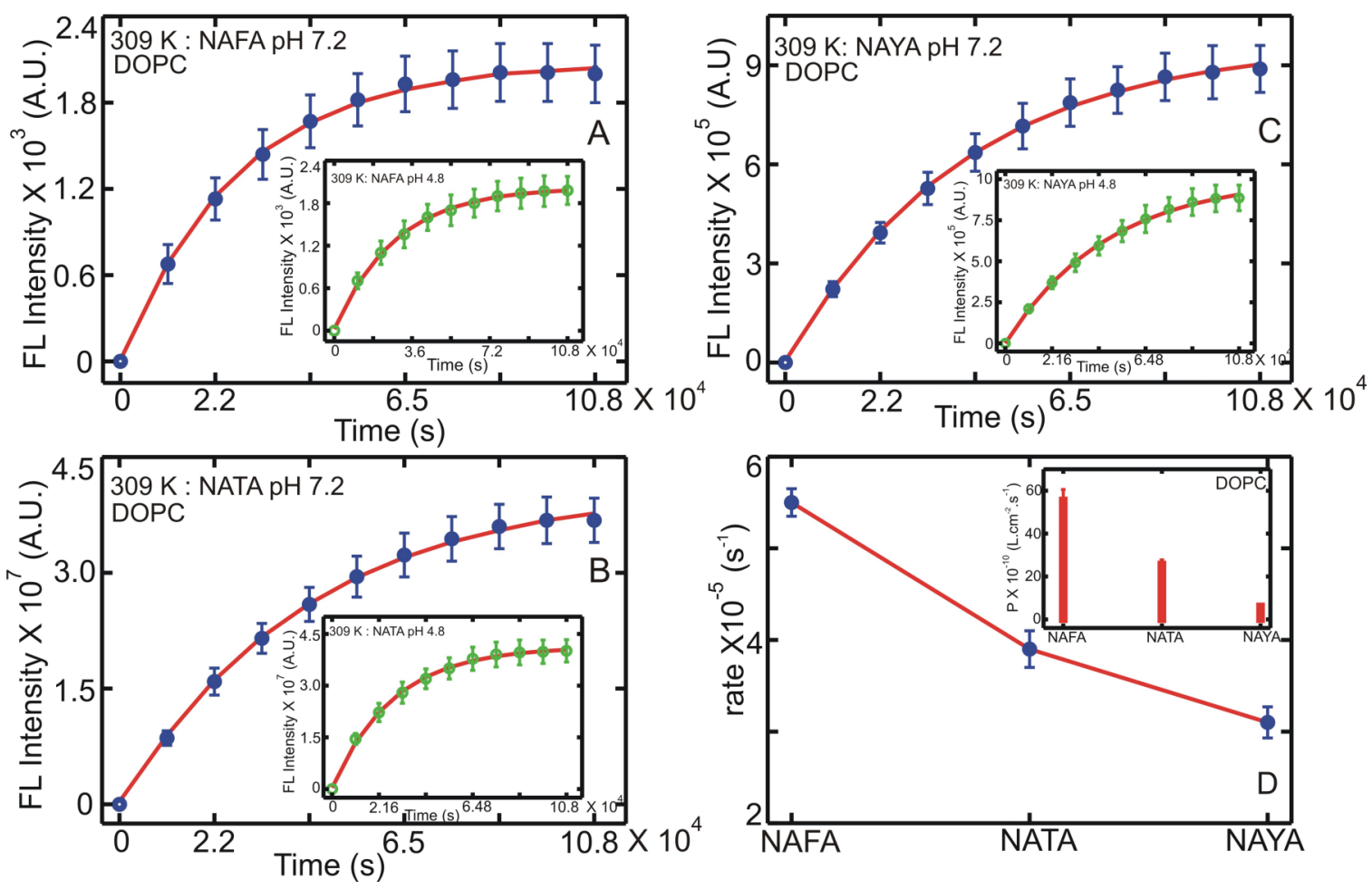

FIG. 2. Experimental results. Integrated fluorescence intensities of three peptides NAFA, NATA, and NAYA at 309 K in DOPC lipid. (a) NAFA, pH 7.2 and inset $\mathrm{pH} 4.8$, (b) NATA, $\mathrm{pH} 7.2$ and inset $\mathrm{pH}$ 4.8, (c) NATA, $\mathrm{pH} 7.2$ and inset $\mathrm{pH} 4.8$, (d) rates of permeation of NAFA, NATA, and NAYA, in inset permeability coefficients of these three peptides, at $309 \mathrm{~K}$ in $\mathrm{pH} 7.2$.

for NAFA, NATA, and NAYA. In the inset of Fig. 2(d), experimentally determined permeability coefficients, $\mathrm{P}$, of the three peptides are presented $\left(\mathrm{P}_{\mathrm{NAFA}}=55 \times 10^{-7} \mathrm{~cm} \mathrm{~s}^{-1}\right.$, $\mathrm{P}_{\mathrm{NATA}}=26 \times 10^{-7} \mathrm{~cm} \mathrm{~s}^{-1}$, and $\mathrm{P}_{\mathrm{NAYA}}=6 \times 10^{-7} \mathrm{~cm} \mathrm{~s}^{-1}$ ). Permeability coefficients are consistent with the observed rate of permeation of all three peptides, with the largest $\mathrm{P}$ value for NAFA and lowest for NAYA. Comparison of measured and calculated permeability coefficients are given in Table I.

The experimental results reported in Figure 2 are in qualitative agreement with previous estimates of Deamer in the sense that our neutral dipeptides permeate membranes much more rapidly than zwitterionic forms of amino acids. ${ }^{82,83}$ However, quantitatively the results are different. The permeation coefficient estimated for the neutral forms of NATA from Deamer's experiments is close to the permeation coefficient of water $\left(\mathrm{P}=10^{-2} \mathrm{~cm} \mathrm{~s}^{-1}\right)$ while our permeation coefficient is considerably slower $\left(\mathrm{P} \sim 10^{-7} \mathrm{~cm}\right.$ $\mathrm{s}^{-1}$ ). It is not clear why the permeation of NATA should be comparable to the permeation of a water molecule. Deamer uses unilamellar vesicles under transmembrane $\mathrm{pH}$ gradients, while our experiments involve planar bilayers and concentration gradients only. Therefore, at least some of the discrepancies can be explained by the differences in experimental setup. We anticipate that more experiments using different experimental techniques will be required to solve this discrepancy.

\section{Computational}

\section{Potentials of mean force}

The potential of mean force represents the relative free energies of a given permeant molecule at different z-distances from membrane center. As seen in Figure 3, all three peptides have qualitatively similar PMFs, exhibiting free energy minima at the lipid-water interface and maxima at the membrane center. The interfacial free energy minima are,

TABLE I. The permeation coefficients and mean passage time for NAFA, NAYA, and NATA. The averages correspond to integration over PMF and $\mathrm{D}(\mathrm{z})$ over the second halves of the US trajectories, while the ranges correspond to results from dividing the data into contiguous quarters.

\begin{tabular}{|c|c|c|c|c|c|}
\hline \multirow[b]{3}{*}{ Molecule } & \multicolumn{3}{|c|}{ Permeation coefficient, $\mathrm{P}\left(\mathrm{cm} \mathrm{s}^{-1}\right)$} & \multirow{2}{*}{\multicolumn{2}{|c|}{$\begin{array}{l}\text { Mean passage time, } \\
\tau(\mu \mathrm{s}) \\
\text { Computational }\end{array}$}} \\
\hline & \multirow[b]{2}{*}{ Experimental } & \multicolumn{2}{|c|}{ Computational } & & \\
\hline & & Average & Range & Average & Range \\
\hline NAFA & $(56 \pm 5) \times 10^{-7}$ & $1 \times 10^{-4}$ & $2 \times 10^{-5}-2 \times 10^{-3}$ & 50 & $30-2600$ \\
\hline NAYA & $(6.2 \pm 1.1) \times 10^{-7}$ & $2 \times 10^{-6}$ & $1 \times 10^{-6}-1 \times 10^{-5}$ & 3000 & $600-3000$ \\
\hline NATA & $(26 \pm 2) \times 10^{-7}$ & $3 \times 10^{-7}$ & $1 \times 10^{-7}-2 \times 10^{-6}$ & 15000 & $3000-30000$ \\
\hline
\end{tabular}




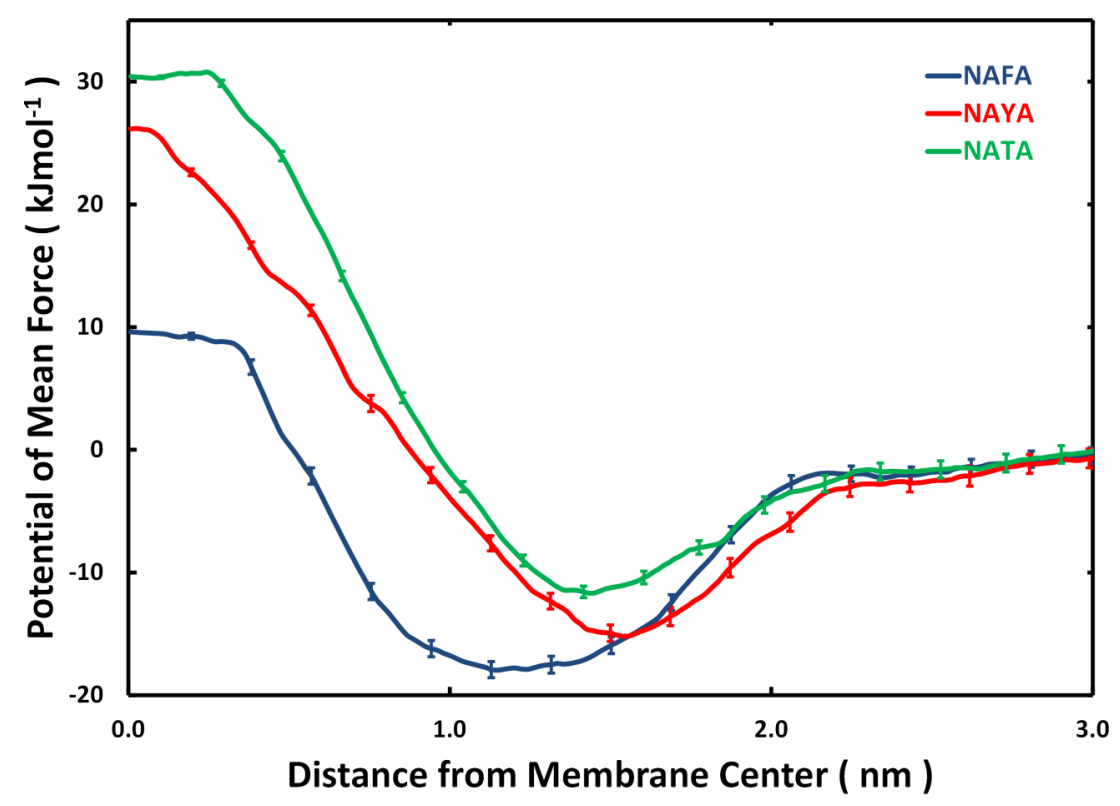

FIG. 3. The potential of mean force is plotted for all three blocked amino acids. The free energy value was set to zero in the solvent region for each data set. The center of the lipid bilayer is located at a $\mathrm{z}=0$. Error bars represent the standard error as calculated by the Bootstrap method. ${ }^{84}$ The standard error was much larger when the calculations were conducted on four contiguous bins of the data: between 3 and $10 \mathrm{~kJ} \mathrm{~mol}^{-1}$ for the central membrane barrier and between 1 and $4 \mathrm{~kJ} \mathrm{~mol}^{-1}$ for the interfacial region (see supplementary material).

respectively, for NAFA, NAYA, and NATA: $-18,-14$, and -12 $\mathrm{kJ} \mathrm{mol}^{-1}$. The central free energy barriers are respectively, for NAFA, NAYA, and NATA: $+28,+41$, and $+44 \mathrm{~kJ} \mathrm{~mol}^{-1}$, relative to the respective minima (and $+10,+27$, and +32 $\mathrm{kJ} \mathrm{mol}^{-1}$ relative to the solution, respectively). The peptide with the most hydrophobic sidechain, NAFA, exhibits the strongest preference for the interface and the lowest central free energy barrier. NAYA and NATA, with partly hydrophilic sidechains, exhibit both weaker binding to the interface and higher barriers in the membrane center. The permeant with the largest sidechain, NATA, has the weakest preference for the interface and the highest barrier. As discussed further in the structural analysis section, the interfacial minima result from a tug of war between hydrophobic and hydrophilic interactions. The most hydrophobic system, NAFA, has the deepest minimum of $-18 \mathrm{~kJ} \mathrm{~mol}^{-1}$ and is located farthest from the membrane center at $1.15 \mathrm{~nm}$. This minimum is also quite broad. For the more polar NAYA and NATA, the minima are shallower $\left(-14\right.$ and $-11 \mathrm{~kJ} \mathrm{~mol}^{-1}$, respectively) and are located closer to the membrane (at 1.54 and $1.45 \mathrm{~nm}$, respectively).

Previous simulations of amino acid sidechains in DOPC by MacCallum et al. predicted interfacial free energies of -13 $\mathrm{kJ} \mathrm{mol}^{-1}$ for phenylalanine and tyrosine, and $-22 \mathrm{~kJ} \mathrm{~mol}^{-1}$ for tryptophan; in the center of the membrane, negative free energies of -5 and $-13 \mathrm{~kJ} \mathrm{~mol}^{-1}$ were respectively predicted for phenylalanine and tryptophan, with a smaller barrier of $7 \mathrm{~kJ} \mathrm{~mol}^{-1}$ for tyrosine. Except for tyrosine, these are qualitatively different from our results, due to the presence of backbone residues in our systems. ${ }^{48}$ Interestingly, our dipeptide PMFs are qualitatively similar to the results of MacCallum et al. for polar sidechains-especially asparagine and glutamine. ${ }^{48}$ Cardenas et al. have studied the permeation of NATA through a DOPC bilayer and found an interfacial minimum of about $-24 \mathrm{~kJ} \mathrm{~mol}^{-1}$ relative to the solution and a barrier of about $75 \mathrm{~kJ} \mathrm{~mol}^{-1}$ relative to the minimum. The quantitative differences from our results are most likely due to their use of the Berger lipid and OPLS/AA protein force fields, as well as a slightly smaller number of DOPC lipids. ${ }^{43,48}$

\section{Translational diffusion}

As described in more detail in the Methods section, we have calculated the diffusion constants using three different approaches-using the numerical solution of the Smoluchowski equation ${ }^{73,74}$ and autocorrelation functions of force fluctuations from separate short trajectories with constrained $\mathrm{z}$ and from force fluctuations of the umbrella restraint force. The first two methods gave consistent results. For a $100 \mathrm{~ns}$ test simulation of NATA in a TIP3P water box with CHARMM36 parameters, the calculated onedimensional NATA center-of-mass diffusion constant was $0.35 \pm 0.04 \times 10^{-9} \mathrm{~m}^{2} \mathrm{~s}^{-1}$, which is in good agreement with the umbrella sampling results at $3.0 \mathrm{~nm}$ of $0.39 \pm 0.01 \times 10^{-9} \mathrm{~m}^{2}$ $\mathrm{s}^{-1}$ from the Smoluchowski equation and $0.26 \pm 0.11 \times 10^{-9}$ $\mathrm{m}^{2} \mathrm{~s}^{-1}$ from constrained MD at $2.0 \mathrm{~nm}$. The translational diffusion estimates based on the umbrella constraint force fluctuations were roughly six times lower than from the first two approaches and were not employed in further analysis. The $\mathrm{D}(\mathrm{z})$ values based on the Smoluchowski equation are presented in Fig. 4. Values obtained from constrained (fixed z) simulations are given in the supplementary material. The diffusion coefficients demonstrate generally little variation with distance from membrane. For NAFA, $D(z)$ changes from $0.44 \times 10^{-9} \mathrm{~m}^{2} \mathrm{~s}^{-1}$ in the solvent region to $0.40 \times 10^{-9} \mathrm{~m}^{2} \mathrm{~s}^{-1}$ at the interface, and to $0.45 \times 10^{-9} \mathrm{~m}^{2} \mathrm{~s}^{-1}$ at the membrane center (except for one outlier of $0.35 \times 10^{-9} \mathrm{~m}^{2} \mathrm{~s}^{-1}$ at $\mathrm{z}=1.8$ $\mathrm{nm})$. Similar effects are seen for NAYA: $\mathrm{D}(\mathrm{z})=0.41 \times 10^{-9}$ $\mathrm{m}^{2} \mathrm{~s}^{-1}$ in the solvent, $0.37 \times 10^{-9} \mathrm{~m}^{2} \mathrm{~s}^{-1}$ at the interface, and $0.44 \times 10^{-9} \mathrm{~m}^{2} \mathrm{~s}^{-1}$ in the center; for NATA: $0.39 \times 10^{-9} \mathrm{~m}^{2}$ $\mathrm{s}^{-1}$ in the solvent, $0.35 \times 10^{-9} \mathrm{~m}^{2} \mathrm{~s}^{-1}$ at the interface, and $0.43 \times 10^{-9} \mathrm{~m}^{2} \mathrm{~s}^{-1}$ in the center. Thus, there is a trend for slower translational diffusion at the interface and for faster diffusion in the center of the lipid bilayer; however, it is weak and barely rises above the statistical uncertainties. There is 


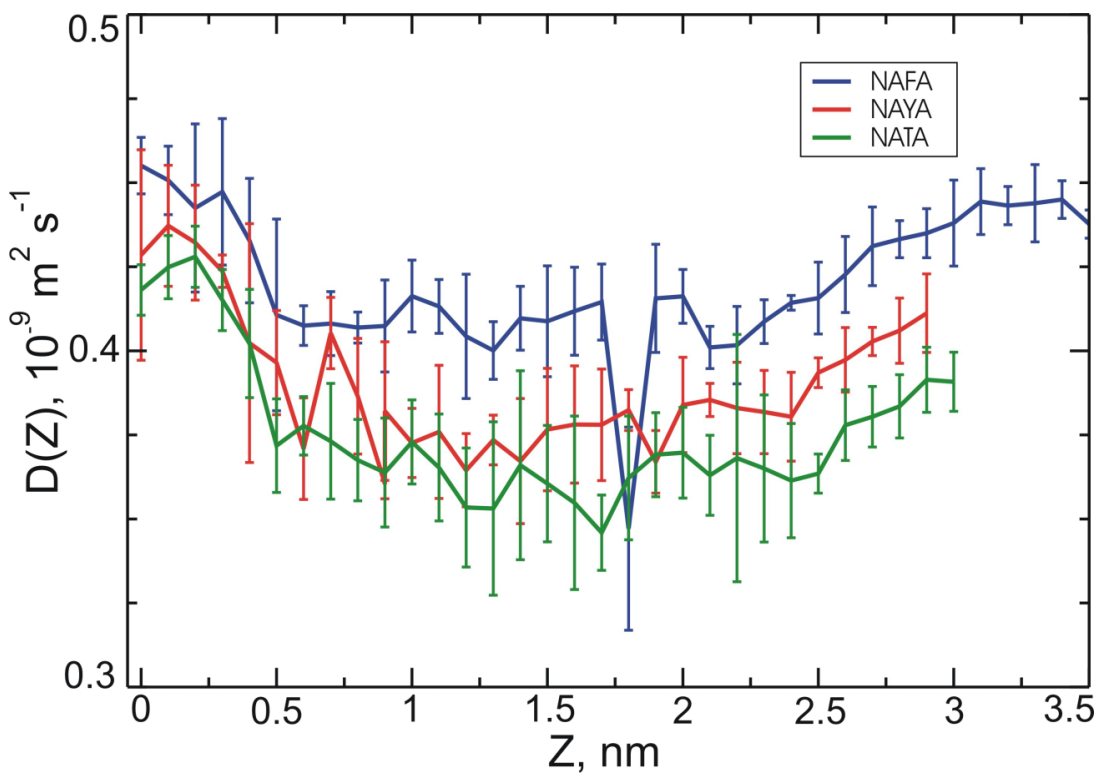

FIG. 4. Translational diffusion constants are plotted as a function of the permeant distance from the center of the lipid bilayer, calculated by numerical solution of the Smoluchowski equation. Error bars were obtained from separate calculations by dividing the data into four contiguous bins.

also a weak systematic trend for diffusion rates between the three peptides, with NAFA > NAYA > NATA, consistent with increasing size. In general, the translational diffusion of the three dipeptides are quite similar, even though their sidechains differ significantly in terms of properties. Why does $\mathrm{D}(\mathrm{z})$ vary so little with respect to membrane insertion, in view of the strong preferential binding of the permeants at the interface and the well-documented lower molecular density in the center of the membrane? Few membrane translocation studies report their diffusion constant data, which reduce our ability to provide insight into this question. Several studies for small molecules do report increased diffusion rates at the center of the lipid bilayer. ${ }^{15,18-21}$ In contrast, most studies of larger molecules that are at least the size of the amino acids studied in this work show relatively flat diffusion profiles in homogenous lipid bilayers. ${ }^{25,27,30}$ Thus, our translational diffusion results are consistent with other reported values for larger molecules.

\section{Permeability measures}

By combining the diffusion coefficients with the PMF profile, quantitative measures of the permeation time scale and rate may be obtained - the mean first passage time (MFPT) and the permeation coefficient, $\mathrm{P}$ (see Methods). Results are reported in Table I. Our simulations predict that NAFA passes through the membrane on a microsecond time scale, whereas NAYA and NATA pass through on a millisecond time scale. The corresponding permeation coefficients are $1 \times 10^{-4}$, $2 \times 10^{-6}$, and $3 \times 10^{-7}, \mathrm{~cm} / \mathrm{s}$ for NAFA, NAYA, and NATA, respectively. The results for tryptophan are quite similar to those for tyrosine, which is to be expected considering their similar diffusion and potential of mean force profiles. Due to the relatively flat diffusion coefficient profiles and the similarity of the $\mathrm{D}(\mathrm{z})$ values for the three peptides, the differences in passive diffusion rates are mostly determined by the free energy profiles. ${ }^{39}$

The calculated passage times are systematically lower and the permeability coefficients are systematically higher than our experimentally measured values for all three peptides. The calculated permeation times differ by many orders of magnitude. Qualitatively, the simulations correctly predict that the permeation of NAFA should be the fastest of the three systems. Quantitatively, the calculated $P$ values for NAYA and NATA of $3 \times 10^{-6}$ and $2 \times 10^{-6}$ $\mathrm{cm} \mathrm{s}^{-1}$, respectively, are comparable to the corresponding experimentally determined results of $6 \times 10^{-7}$, and $26 \times 10^{-7}$ $\mathrm{cm} \mathrm{s}^{-1}$, respectively. However, this order is reversed in the simulations. Although a direct comparison does not exist for phenylalanine and tyrosine, our results are roughly the same order of magnitude as those obtained using the same model for small molecules. ${ }^{25}$ Previous studies of tryptophan using the more advanced method of milestoning report permeation times on the time scale of hours, which are also in agreement with experimental results. ${ }^{43,44,85,86}$ Most other studies have also reported much larger and faster permeability coefficients using the inhomogeneous solubilitydiffusion model, which has been discussed in great detail in other works. ${ }^{18,25,43,44,87-89}$ Several assumptions underpin the solubility-diffusion model, including memoryless, diffusivetype motion along the reaction coordinate and the presence of only one slow variable describing the motion of the permeant-namely, the translocation along the membrane normal. ${ }^{15,89}$ Many recent studies suggest that an additional rotational barrier exists that slows down the movement of the permeant. ${ }^{18,43,44,87,89,90}$ Others hypothesize that membrane and solvent structural fluctuations play an important role as well. ${ }^{39,85}$ The CHARMM lipid force field also overestimates the electric field strength within the membrane by a factor of 3 , which may be lowering the value of the PMF within the membrane interior and subsequently accelerating the mean passage time. ${ }^{63}$

The discrepancy between our experimental and theoretical results can be attributed to any or all of the above concerns. Additional slow variables such as rotational barriers or membrane fluctuations can couple with the longitudinal translocation of the permeant and slow down the process. For example, Fig. 6(b) demonstrates that NAYA adopts 
preferential rotational orientations based upon its depth within the membrane. The correlation of these motions could not be calculated within the time frame of each umbrella window. These motions may be essential for the longitudinal diffusion of the permeant, but they are not included within our permeability model. This phenomenon has been well documented in assisted diffusion and passive diffusion through channels formed by porins. ${ }^{91}$ For NATA, rotational barriers have also been found to reduce the permeability coefficient through more advanced models such as milestoning. ${ }^{85,89}$ Similarly, membrane fluctuations within the lipid bilayer also play a role in the permeation process. For small molecules, these fluctuations are very fast, with time scales less than a nanosecond. ${ }^{92}$ For larger molecules, membrane effects become increasingly more important. As Neale et al. discovered, there are rare sampling barriers at the lipid interface with time scales on the order of $10 \mu \mathrm{s}$-far too long for our simulations to detect. ${ }^{93}$ Whether our amino acids fall into the small or large category remains unknown and would require much longer simulation times. Finally, the increased electric field strength within the membrane may be assisting our amino acids to adopt favorable orientations on a much faster time scale due to the increased forces on the molecular dipole. All of these concerns would cause our theoretical permeability coefficients to be far larger than those determined experimentally.

\section{Rotational diffusion}

Rotational correlation times $\tau_{\text {rot }}$ for the peptide sidechains are presented in Fig. 5. Unlike the translational diffusion rates, rotational diffusion speeds change very strongly upon membrane insertion. Rotations are fastest in the aqueous region, with average sidechain $\tau_{\text {rot }}$ values of 17,33 , and 27 ps for NAFA, NAYA, and NATA, respectively. Reorientational motions slow down dramatically as the peptide approaches the lipid-water interface, with average $\tau_{\text {rot }}$ values of over 0.3 to $1.5 \mathrm{~ns}$ for all three peptides at $\mathrm{z}=2 \mathrm{~nm}$. The reorientation rates are even slower in the headgroup region, with $\tau_{\text {rot }}$ in the 1-4 ns range for $\mathrm{z}=0.5-1.3 \mathrm{~nm}$. Motions in the bilayer center occur at rates intermediate between water and headgroups, with $\tau_{\text {rot }}=300$ ps, 800 ps, and 200 ps for NAFA, NAYA, and NATA, respectively. If the effects of local viscosity changes were the dominant effect, we would expect a similar variation of translation diffusion, $\mathrm{D}(\mathrm{z})$, and rotational correlation $\tau_{\text {rot }}(\mathrm{z})$ with membrane insertion. However, because the translational diffusion profile is quite flat, the variations of $\tau_{\text {rot }}$ by factors 10-100 must result from very strong specific interactions with the lipid environment. Clear evidence for strong preferential binding of the peptides with the interface is seen in the PMF plots in Fig. 1. What is unexpected is the slowing down of reorientations by as much as a factor of 100 in the headgroup region and by 10 in the membrane center,
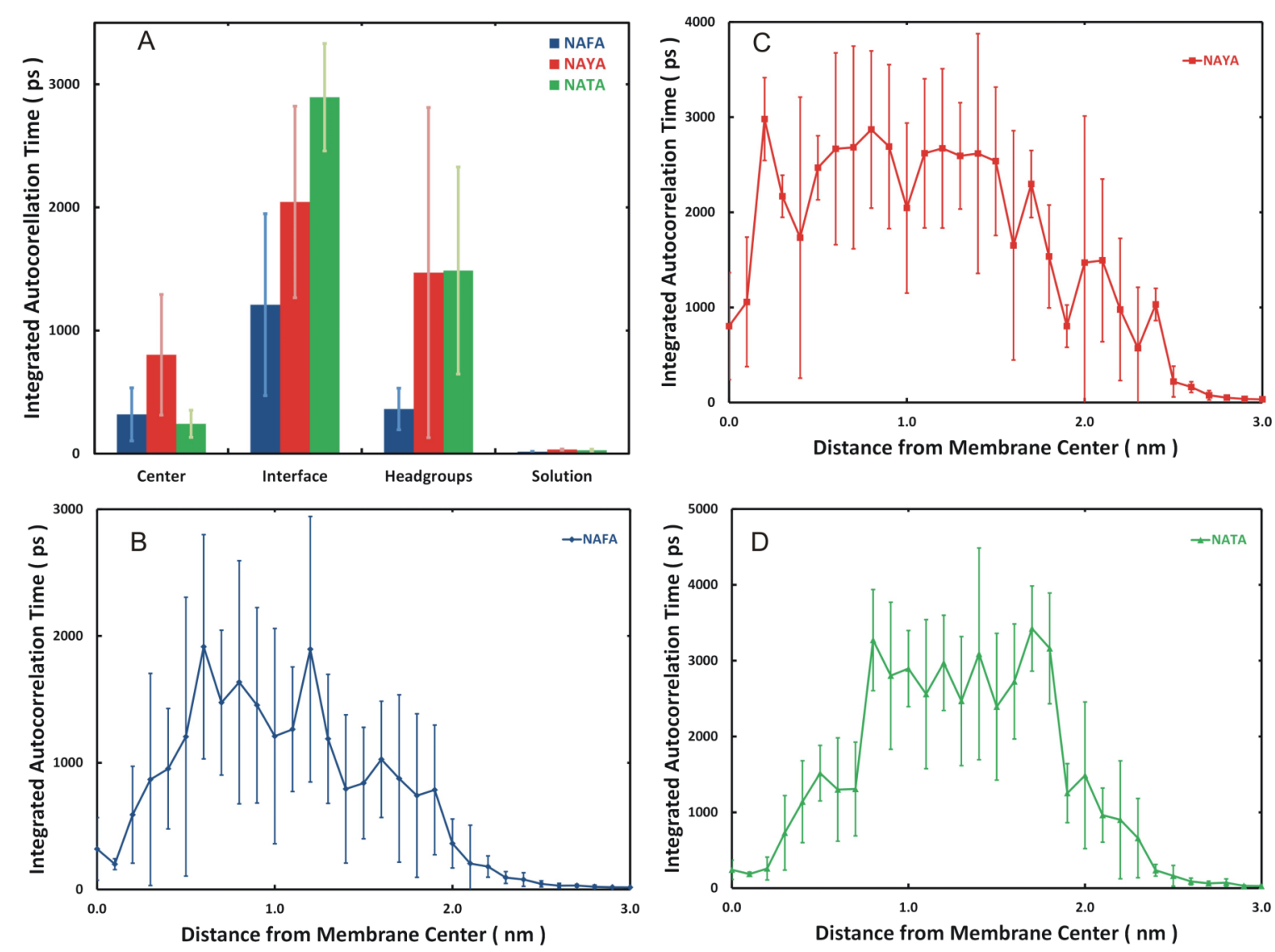

FIG. 5. Sidechain rotational correlation times as a function of the membrane insertion distance, $\mathrm{z}$. (a) Comparison of $\tau_{\text {rot }}$ values at $\mathrm{z}=0,1.0,2.0$, and 3.0 nm for the three peptides and values of $\tau_{\text {rot }}(\mathrm{z})$ for NAFA (b), NAYA (c), and NATA (d). Values obtained by integrating the autocorrelation function for the transition axis of each of the sidechains (see Methods). In the headgroup region, from 0.5 to $1.5 \mathrm{~nm}$, the estimated correlation times are approximate because in many cases the autocorrelation functions do not decay to zero, denoting incomplete sampling in the trajectories. 
A

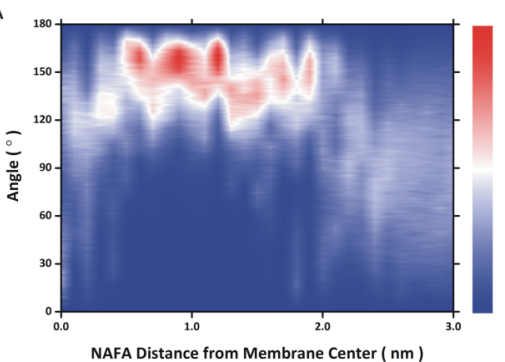

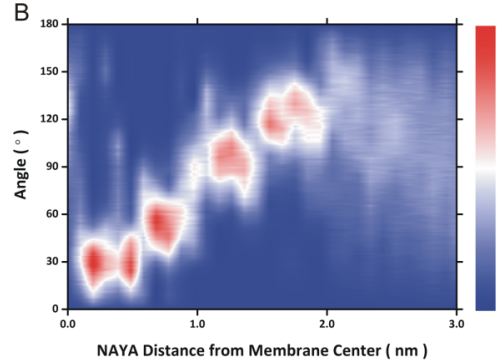

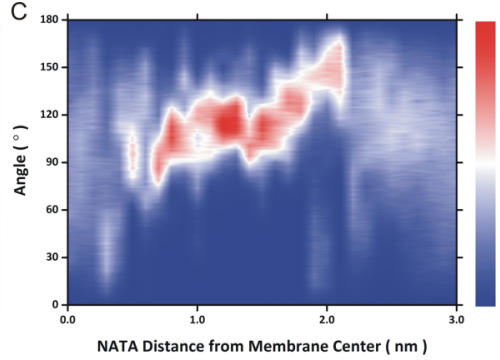

FIG. 6. Insertion angles $\theta$. A vector is defined from the center of mass of the backbone to the center of mass of the sidechain. The angle $\theta$ is between this vector and the positive $\mathrm{z}$-axis of the simulation box. The normalized probability density, $\mathrm{P}(\theta, \mathrm{z})$, is plotted with blue representing little or no probability and with red representing the highest probability.

compared to solution. Previous studies, which focused on translational dynamics, typically found faster motions in the central region of lower relative density. ${ }^{15,18-21}$ Presence of slow reorientations in the course of membrane transport has been previously explored..$^{25,27,30}$

Our simulations suggest that translational diffusion in the $\mathrm{z}$ direction, along the elongated lipid molecules, is relatively easy; while rotational diffusion of the peptide sidechains, which are tethered to their backbones and must move in directions perpendicular to the tightly packed lipids, is unexpectedly slow. Our results present a new look at peptide dynamics in membranes, indicating that computational modeling and experimental measurement of reorientational motions should be a sensitive probe of peptidemembrane interactions. A more detailed analysis of peptidelipid interactions is given below (see Specific interactions).

Computer simulations of NAFA, NAYA, and NATA reorientations using the CHARMM force field and TIP3P water have reported values of $\tau_{\text {rot }}=20,27$ and $30 \mathrm{ps,}$ respectively, at $298 \mathrm{~K}^{81}$ Experimental values reported previously were $40 \pm 5$ ps for NAYA and $48 \pm 5$ ps for NATA at $298 \mathrm{~K}$, and $98 \pm 30$ ps for NAFA at $278 \mathrm{~K} \cdot{ }^{81}$ Our aqueous region $\tau_{\text {rot }}$ values are in very good agreement with the previous calculations and also in good agreement with the experimental estimates at $298 \mathrm{~K}$, given that the TIP3P water model systematically underestimates the viscosity of water. ${ }^{65}$

\section{Insertion angle}

The peptide insertion angle, $\theta$, is defined as the angle between the $\mathrm{z}$ axis and the vector pointing from the center-ofmass $(\mathrm{COM})$ of the backbone to the COM of the sidechain.
The distribution of these angles is plotted in Figure 6. All three simulated peptides exhibit a wide range of allowed angles in the aqueous phase, corresponding to free reorientations. ${ }^{48}$ At $\mathrm{z}=2.2 \mathrm{~nm}$ for tryptophan and $2.0 \mathrm{~nm}$ for phenylalanine and tyrosine, reorientations become restricted. Nearing the headgroup region in the $\mathrm{z}=1.5-2.0 \mathrm{~nm}$ range, all three peptides insert at an angle of around $150^{\circ}$, corresponding to the backbone pointing into the solvent and to the sidechain pointing into the lipid headgroups. For NAFA, this insertion angle remains stable at the preferred value until $\mathrm{z}=0.5 \mathrm{~nm}$, after which a broad distribution of insertion angles reappears in the membrane center in the $\mathrm{z}=0.0-0.5 \mathrm{~nm}$ range. NAYA undergoes a systematic change in the preferred insertion angle while permeating into the membrane. The angle changes to $90^{\circ}$ at $\mathrm{z}=1.5 \mathrm{~nm}, 60^{\circ}$ at $\mathrm{z}=0.8 \mathrm{~nm}, 30^{\circ}$ for $\mathrm{z}$ in the $0.1-0.6$ $\mathrm{nm}$ range, and finally rotates freely at the center. For NATA, the insertion angle changes in a manner somewhere between the NAFA and NAYA cases. In NATA, the preferred insertion angle changes to $120^{\circ}$ for $\mathrm{z}$ between 1.0 and $2.0 \mathrm{~nm}$ and to about $90^{\circ}$ for $\mathrm{z}$ between 0.5 and $0.8 \mathrm{~nm}$, with mostly unrestricted orientations within the $\mathrm{z}=0.0-0.5 \mathrm{~nm}$ range. NATA's ability to reorient in the central membrane region, as found in our simulations, agrees with the results obtained previously by umbrella sampling and milestoning from other groups. ${ }^{43,89}$

In general, all three aromatic dipeptides tend to initiate interactions with the membrane by orienting their sidechains toward the lipid headgroups and backbones towards the solution. The most hydrophobic peptide, NAFA, retains this orientation throughout the headgroup region, while NAYA and NATA assume an orientation parallel to membrane surface at the interface. Finally, at the center of the membrane, the
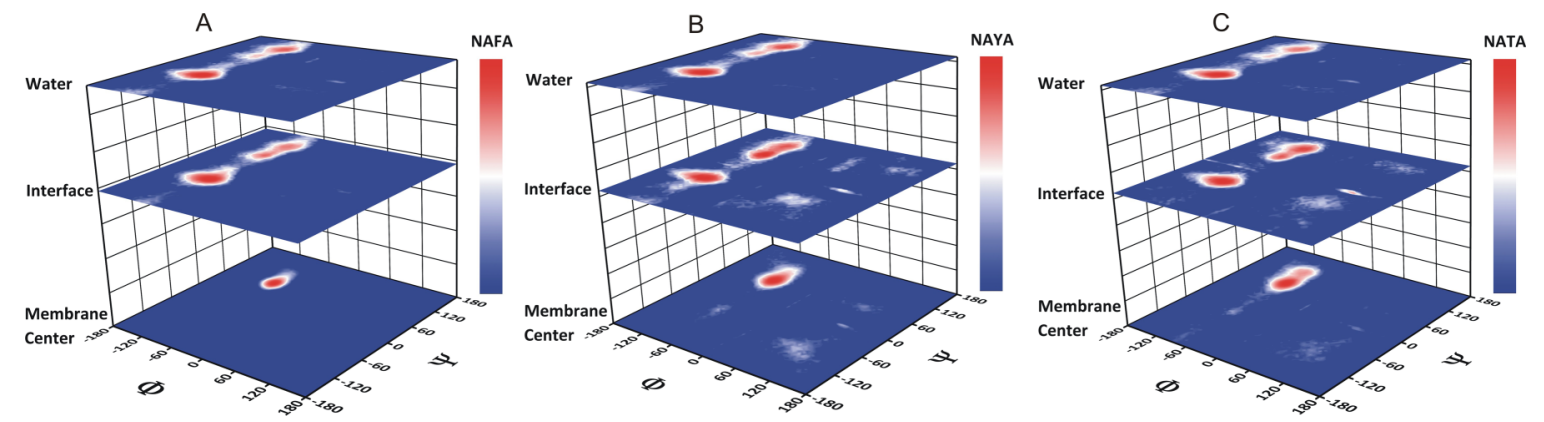

FIG. 7. Ramachandran plots, in terms of the natural log of the probability density, are presented above for (a) NAFA, (b) NAYA, and (c) NATA at the center of the lipid bilayer, $\mathrm{z}=0$ (bottom), the interfacial region, $\mathrm{z}=1.5 \mathrm{~nm}$ (middle), and the aqueous region, $\mathrm{z}=3.0 \mathrm{~nm}$. 

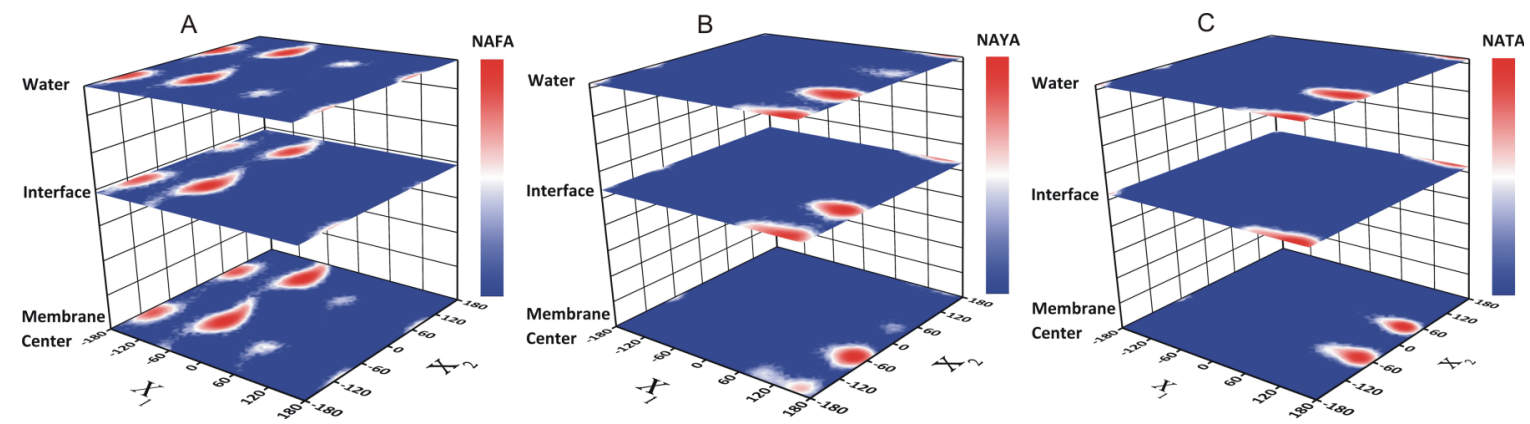

FIG. 8. Peptide sidechain conformations as function of membrane insertion z. Natural log of population for probability of $\left(\chi_{1}, \chi_{2}\right)$ distributions for (a) NAFA, (b) NAYA, and (c) NATA.

most hydrophilic peptide, NAYA, reorients to a direction that is almost opposite of its value in solution, before again assuming free rotation at $\mathrm{z}=0.0 \mathrm{~nm}$. In contrast, the orientation of the more hydrophobic peptides, NAFA and NATA, is unrestricted over a wider range of positions, $0.0-0.5 \mathrm{~nm}$. This difference in behavior may possibly be the result of water molecules pulled into the membrane's interior by the permeating peptides (see Preferential Interactions).

\section{Backbone conformations}

Ramachandran plots representing peptide backbone conformations are presented in Fig. $7 .{ }^{94}$ In the aqueous region of the simulation box, the $\phi$ and $\psi$ angles of NAFA, NAYA, and NATA are grouped into three areas: the large region with negative $\psi$ angles represents $\alpha$-helical type conformations, the region in the top left corner- $\beta$-sheet and extended structures, and the small region in-between the $\mathrm{C}_{7 \mathrm{eq}}$ conformer characteristic of dipeptides. ${ }^{95}$ As the dipeptides move from solution to interface, the probabilities in the $\alpha$ and $\beta$ regions decrease, while that of $\mathrm{C}_{7 \mathrm{eq}}$ increases. For all three dipeptides, $\mathrm{C}_{7 \mathrm{eq}}$ becomes the dominant structure at the center of the membrane. The three peptides thus exhibit a clear structural response to the different chemical environments in solution, membrane interface, and center, which is a very exciting result. The role of such conformational
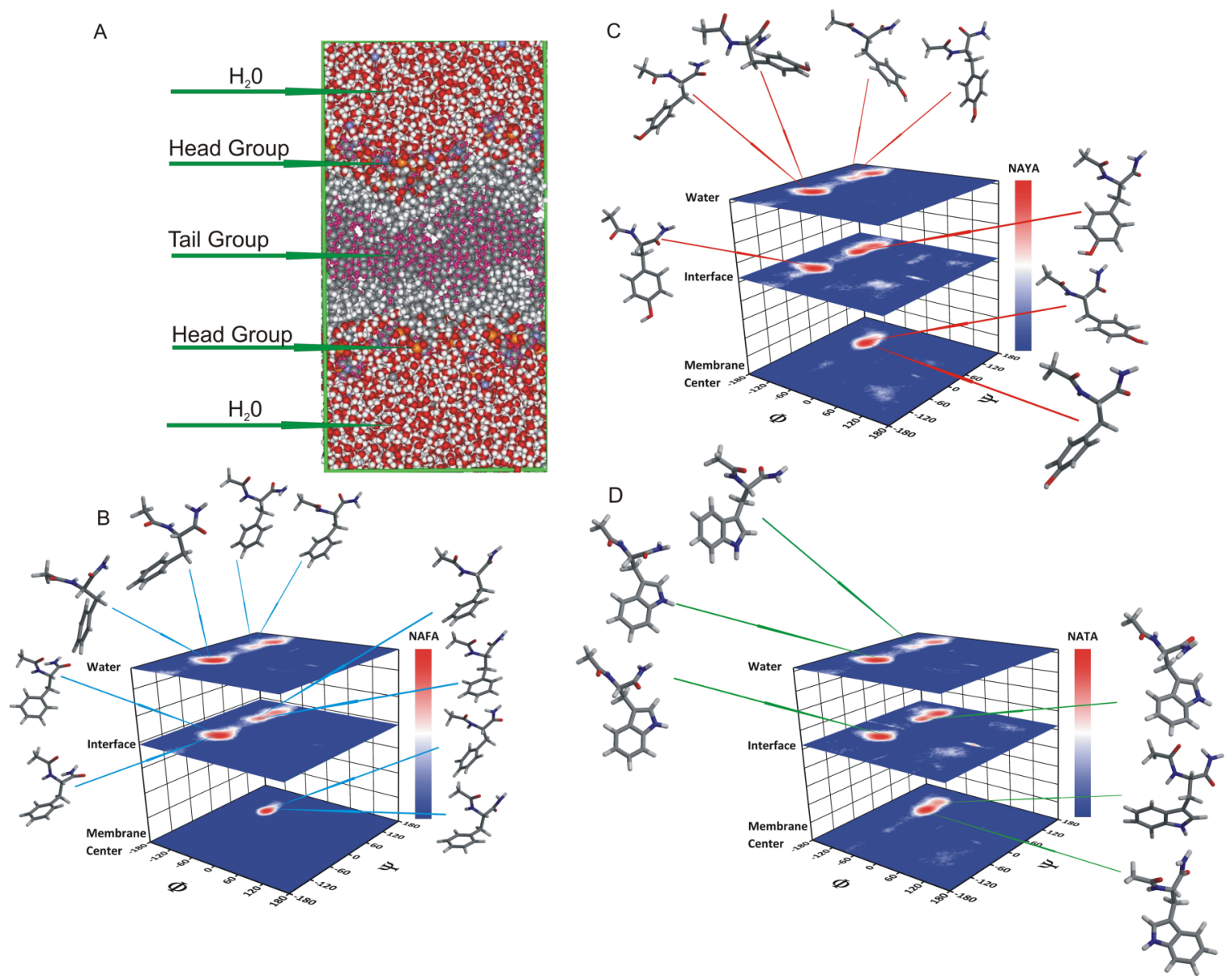

FIG. 9. Shown are representative structures from trajectory clustering. Lipid bilayer with water molecules, hydrophilic headgroups, and hydrophobic tail group shown in (a). Central structures of structural clusters corresponding to the alpha and $\mathrm{C}_{7 \text { eq }}$ free energy minima are shown, in (b) NAFA, in (c) NAYA, and in (d) NATA. 


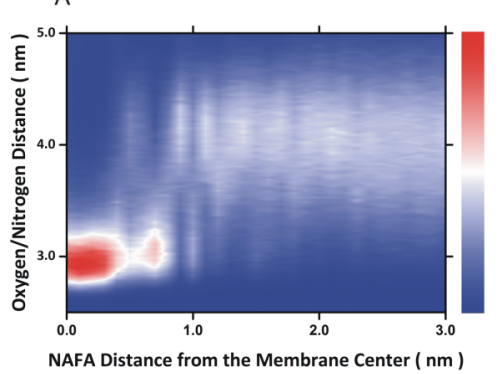

B

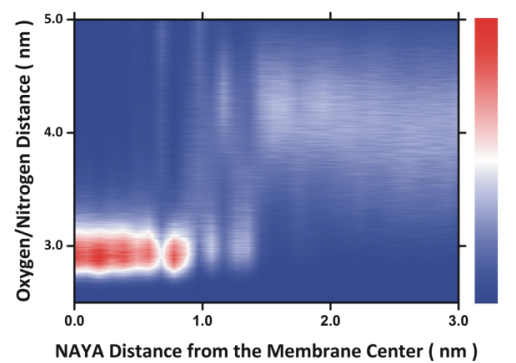

$\mathrm{C}$

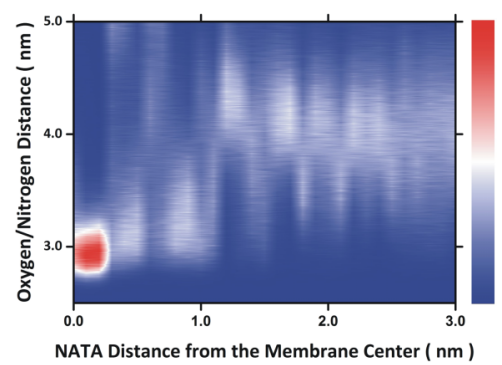

FIG. 10. Distributions of O $\cdots \mathrm{N}$ distances between blocking groups in (a) NAFA, (b) NAYA, and (c) NATA peptides as function of distance from membrane center. Figures show probability distributions $\mathrm{P}\left(\mathrm{R}_{\mathrm{NO}}, \mathrm{z}\right)$.

change in more complicated structures is worthy of further investigation.

\section{Sidechain conformations}

Distributions of the sidechain angles $\left(\chi_{1}, \chi_{2}\right)$ for each peptide were also examined (Figure 8 ). The nonpolar phenyl ring of NAFA sampled the same four main conformers along the whole permeation path $\left(\mathrm{tg}^{-}, \mathrm{tg}^{+}, \mathrm{g}^{-} \mathrm{g}^{-}, \mathrm{g}^{-} \mathrm{g}^{+}\right)$. The phenol ring of NAYA sampled two main sidechain conformers along the whole path $\left(\mathrm{tt}, \mathrm{tg}^{-}\right)$. The indole ring of NATA explored two main conformers in solution $\left(\mathrm{tt}, \mathrm{tg}^{-}\right)$, one at the interface (tt) and two in the bilayer center $\left(\operatorname{tg}^{-}, \operatorname{tg}+\right)$. Thus, the more polar phenol and indole exhibited different conformational preferences than the nonpolar phenyl.

\section{Peptide structures-Clustering}

Clustering of the trajectories in dihedral angle space confirmed that the studied peptides undergo definite structural changes during membrane permeation. Examples of main sampled conformations are shown in Fig. 9. For NAFA, seven clusters were found in the solution region, with 4 corresponding to $\alpha, 1$ to $\beta$, and 3 to $C_{7 \text { eq }}$ structures; at the interface four clusters were identified, $2 \alpha$ and $2 \mathrm{C}_{7 \text { eq }}$ and in the membrane center four clusters of $\mathrm{C}_{7 \text { eq }}$ type were sampled, with different sidechain combinations. For NAYA, 37 total clusters were found in solution, of which 8 had populations above $0.5 \%-4 \alpha, 1 \beta$, and $3 \mathrm{C}_{7 \text { eq }}$. At the interface, NAYA sampled only two clusters, one in $\alpha$ and one in $\mathrm{C}_{7 \text { eq }}$ region, and in membrane center 3 clusters were sampled, all of $\mathrm{C}_{7 \text { eq }}$ type. For NATA, the situation was the simplest-there were only two clusters in each environment $-1 \alpha$ and $1 \mathrm{C}_{7 \text { eq }}$ in solution and at interface, and two $\mathrm{C}_{7 \text { eq }}$ in membrane center. The growing population of the $\mathrm{C}_{7 \text { eq }}$ conformation upon membrane insertion may be illustrated by the changes in the $\mathrm{O} \cdots \mathrm{N}$ distance between carbonyl oxygen of the N-terminal blocking group and the nitrogen of the C-terminal blocking group shown in Fig. 10. In solution, this distance fluctuates over a relatively wide range of values, $3-5 \mathrm{~nm}$, while a narrow range of 2.8-3.1 nm is sampled inside the membrane. Thus, in the hydrophobic environment of the lipid acyl chains, the peptide forms a self-interaction, partially shielding polar backbone atoms from the external medium.

\section{Molecule shape and size}

Changes of the radius of gyration $\left(R_{g}\right)$ and solvent accessible surface area (SASA) with membrane insertion is shown in the supplementary material. The peptide size,

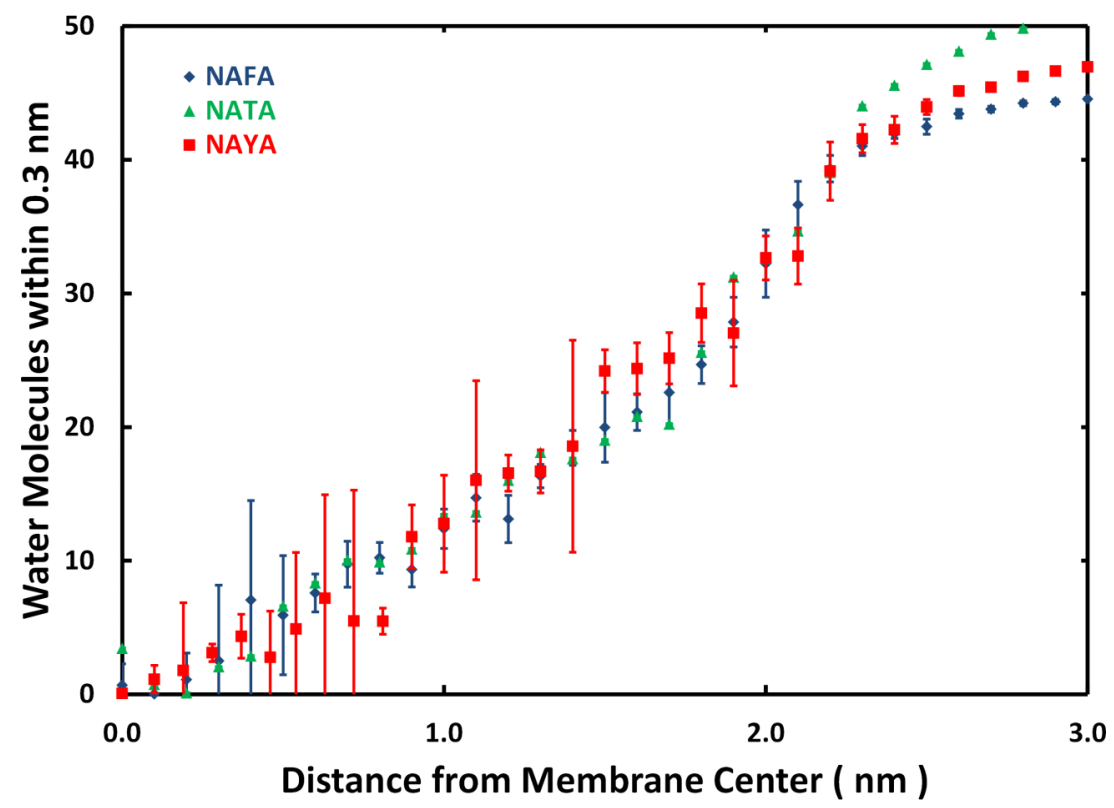

FIG. 11. Average number of water molecules within $0.3 \mathrm{~nm}$ of the peptides. 

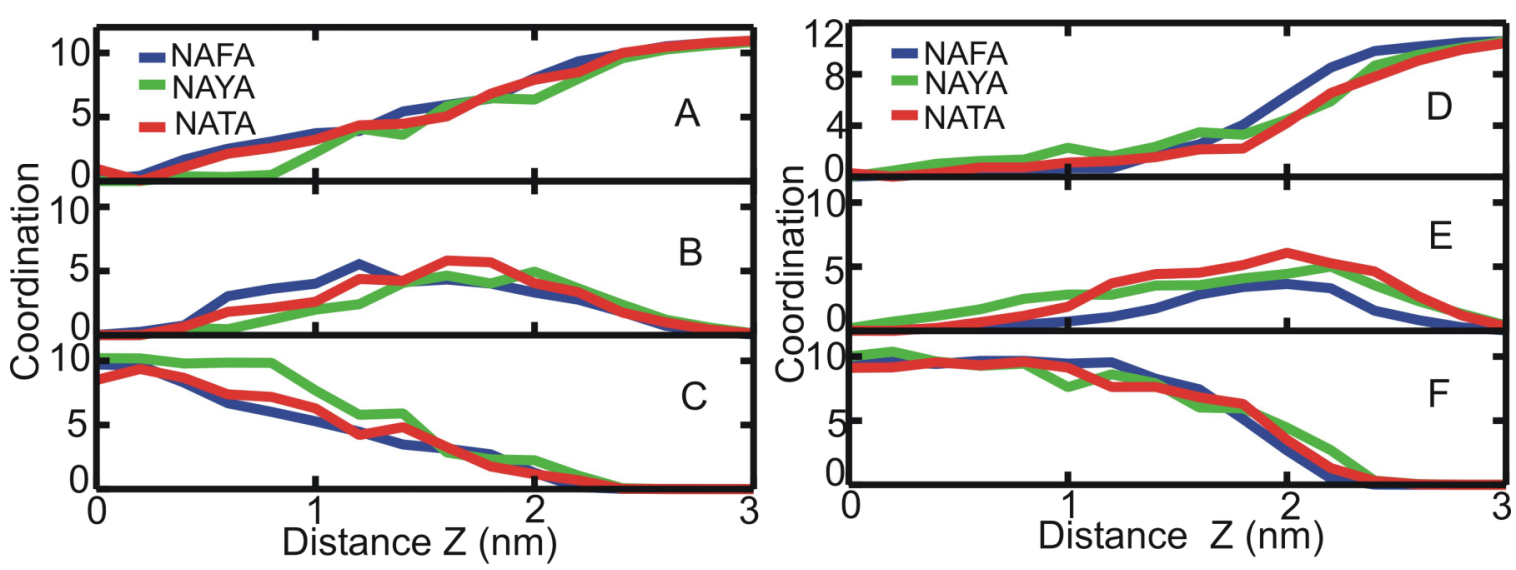

FIG. 12. Average coordination numbers of the three peptides as a function of insertion depth z. Coordination of backbone atoms of peptides by (a) water, (b) headgroups, and (c) lipid tails. Coordination of sidechain atoms of peptides by (d) water, (e) headgroups, and (f) lipid tails. The presented X-Y values are the average number of atoms of species $\mathrm{Y}$ within $0.5 \mathrm{~nm}$ of an atom of species X. Species are as follows: backbone-non-hydrogen atoms of peptide backbone and blocking groups; sidechains—-non-hydrogen atoms of peptide sidechains; water-water oxygens; tails—lipid acyl tail carbon atoms; heads—headgroups, non-hydrogen atoms of lipids, excluding tails.

measured by $\mathrm{R}_{\mathrm{g}}$, and the solvent exposure, measured by SASA, do not exhibit significant variation as a function of membrane insertion. Only in the case of NATA, a small effect of slight lowering of $\mathrm{R}_{\mathrm{g}}$ and SASA is found in the tail region, $\mathrm{z}=0.0-0.5 \mathrm{~nm}$. Interestingly, the conformational changes observed for the peptides as they translocate across the membrane have little effect on SASA and $R_{g}$ for these relatively small systems.

\section{Specific interactions}

Interactions between the peptides and their environment are analyzed below. Figure 11 shows the average number of water molecules within $0.3 \mathrm{~nm}$ of the peptide as a function of $\mathrm{z}$, representing roughly the first solvation shell. Peptides were solvated by 45-50 waters in the aqueous region. The number of waters decreased systematically with insertion depth, reaching ca. 20 at the interface $(\mathrm{z}=1.5 \mathrm{~nm})$, ca. 15 at $\mathrm{z}=1.0 \mathrm{~nm}$, and ca. 5 at $z=0.5 \mathrm{~nm}$. All peptides pull a large number of water molecules with them as they enter the membrane. Even at the center, $\mathrm{z}=0$, residual solvation remains: $0.7,0.1$, and 3.4 on average for NAFA, NAYA, and NATA, respectively. Previous simulations of NATA in DOPC found 4 waters solvating the peptide in membrane center, very close to our result. ${ }^{43}$

Figure 12 shows the details on specific interactions of the peptides and the lipids. The average coordination numbers show the expected trends - upon insertion into the membrane, the number of solvating waters systematically decreases, the number of acyl carbons increases, and the number of headgroup atoms first increases to a maximum in the interfacial region and then drops off. For the backbone (Figs. 12(a)-12(c)), there are some interesting differences between the three peptides. The highest backbone coordination by headgroups occurs at $\mathrm{z}=1.2 \mathrm{~nm}$ for NAFA, $2.0 \mathrm{~nm}$ for NAYA, and $1.6 \mathrm{~nm}$ for NATA. For NAYA, the coordination by lipid tails is systematically greater and coordination by water systematically lower than for the other peptides. The switchover distance, at which the coordination by water and acyl tails becomes equal, is at 1.2, 1.6, and $1.4 \mathrm{~nm}$ for NAFA, NAYA, and NATA backbone, respectively. The behavior of the peptide sidechains (Figs. 12(d)-12(f)) is similar to the backbone. Highest sidechain coordination by headgroups occurs at $\mathrm{z}=2.0 \mathrm{~nm}$ for NAFA, $2.2 \mathrm{~nm}$ for NAYA, and $2.0 \mathrm{~nm}$ for NATA. This is consistent with the membrane insertion angles discussed above, with NAFA inserting mostly sidechain-first, NAYA mostly at a $90^{\circ}$ "sideways" angle, with both sidechain and backbone, and NATA intermediate between the other two peptides. All three peptides appear to be able to interact well with the lipid tails, reaching tail coordination numbers at $\mathrm{z}=0$ of similar value to those by water at $\mathrm{z}=3.0 \mathrm{~nm}$.

\section{CONCLUSIONS}

We present the results of a joint experimental and computational study of the passive permeation of three aromatic dipeptides-NAFA, NAYA, and NATA - through DOPC lipid bilayers. In the experimental part, permeation times and permeability coefficients were measured for the three peptides under physiological conditions ( $\mathrm{pH} 7.2$ and 309 $\mathrm{K}$ ) showing that NAFA exhibits fastest and NAYA the slowest translocation rates. The experimental permeation times were in the 5-10 $\mathrm{h}$ range for the three peptides studied. At a lower $\mathrm{pH}$ of 4.8 , measurements showed faster permeation by NAYA and NATA, and slower by NAFA. In the computational part, we performed umbrella sampling simulations for the three systems, using at least 30 windows of 50-100 ns length for each peptide to model the thermodynamics, dynamics, and microscopic interactions along the chosen one-dimensional reaction path $\mathrm{z}$, the center-of-mass distance between peptide and lipid bilayer. The calculated profiles of the potential of mean force show two strong effects-preferential binding of each of the three peptides to the lipid interface, with free energies of $-18 \mathrm{~kJ} / \mathrm{mol}$ of NAFA, $-14 \mathrm{~kJ} / \mathrm{mol}$ for NAYA and $-12 \mathrm{~kJ} / \mathrm{mol}$ for NATA and large free energy barriers in the membrane center $+28 \mathrm{~kJ} / \mathrm{mol}$ for NAFA, $+41 \mathrm{~kJ} / \mathrm{mol}$ for NAYA and $+44 \mathrm{~kJ} / \mathrm{mol}$ for NATA, relative to the respective minima. We use three approaches to calculate the positiondependent translational diffusion coefficients $\mathrm{D}(\mathrm{z})$. Of these, 
methods based on numerical solution the Smoluchowski equation and on force autocorrelations from short trajectories with constrained values of $\mathrm{z}$ give consistent results, agreeing with independent estimates in the solution phase, while use of autocorrelations of the restraint force significantly overestimates $D(z)$ values. Surprisingly, computed $D(z)$ values change very little with reaction coordinate and are also quite similar for the three peptides studied. In contrast calculated values of sidechain rotational correlation times $\tau_{\text {rot }}(\mathrm{z})$ show extremely large changes with peptide membrane insertionvalues become 100 times larger in headgroup region and 10 times larger in membrane center, relative to solution. Thus, it appears that these small peptides can relatively easily undergo translational diffusion along the $\mathrm{z}$ axis, parallel to the lipid molecules, while reorientations, involving motion perpendicular to the lipids, is strongly hindered, especially in the tightly packed headgroup region.

Analysis of the insertion angle shows the peptides inserting initially with the sidechain pointing into the membrane and backbone into solution. While NAFA retains this preferred orientation through the interfacial and headgroup regions, NAYA systematically switches to a backbone-in orientation as its insertion progresses, while NATA behaves in an intermediate fashion, changing to a perpendicular orientation. In the central region of the membrane all three peptides sample the full range of insertion angles. Another interesting feature of the simulated peptide permeation is the conformational change. The peptide conformational freedom becomes systematically restricted as they enter the membrane: $\alpha, \beta$, and $\mathrm{C}_{7 \text { eq }}$ basins are explored in solution, $\alpha$ and $\mathrm{C}_{7 \text { eq }}$ at the interface and only $\mathrm{C}_{7 \text { eq }}$ in the center. The $\mathrm{C}_{7 \mathrm{eq}}$ structures are characterized by a short contact between the polar atoms of the blocking groups. Analysis of peptide interactions with the environment showed that in the process of permeation the peptide interactions with water are replaced first by the lipid headgroups and then by the lipid sidechains. The passage of sidechain and backbone through the different regions is consistent with the insertion angle analysis. Some residual waters of solvation remain even in the membrane center, deforming the membrane structure, as previously noted. ${ }^{43}$

The experiments described in this work provide useful baseline information for aromatic peptide membrane permeation processes-passage times and permeation coefficients. Our calculated values of passage times are several orders of magnitude smaller than experimental data, while permeation coefficients for NATA and NAYA are in reasonable agreement. This effect has been found in previous studies, and has been attributed primarily to the presence of more than one slow variable characterizing membrane permeation, among which peptide orientation and large-scale membrane structural fluctuations have been proposed. ${ }^{25,27,30,96}$ The accommodation of additional slow variables is an exciting topic of current inquiry.

Excluding the passage times and permeation coefficients, our simulation results are in reasonable agreement with most studies on similar systems, including computer simulations with different force fields and experimental measurements. Our PMF profile for NATA qualitatively agrees with results of Cardenas et al., ${ }^{43}$ and the calculated translational and rotational diffusion rates in the solution region agree with both computational results and experimental data. $15,18-21,25,27,30$ Thus, we believe that our combined experimental and computational study provides improved understanding of the process of transmembrane permeation of small aromatic peptides. Especially valuable are the microscopic insights from the simulations, including the large difference between translational and rotational diffusion rates and changes in peptide structure as a function of membrane insertion depth. Membrane permeation by flexible amphiphilic molecules remains a fruitful area for further studies.

\section{SUPPLEMENTARY MATERIAL}

Additional simulation details are available in the supplementary material.

\section{ACKNOWLEDGMENTS}

Use of computational resources at the Advanced Computing Facility at the University of Kansas, supported by NSF MRI Grant No. 1337899 is gratefully acknowledged. We acknowledge partial support for local computing resources from the University of Kansas General Research Fund and partial support from a Walrafen Fellowship from the Department of Chemistry, University of Kansas (B.L.L.). G.S.J. would like to thank Wilson R. Veras Tavarez and Elizabeth De Leon Olmeda of UCC for helpful comments.

${ }^{1}$ D. W. Deamer, Nature 454(7200), 37-38 (2008).

${ }^{2}$ K. Adamala and J. W. Szostak, Nat. Chem. 5(6), 495-501 (2013).

${ }^{3}$ J. P. Overington, B. Al-Lazikani, and A. L. Hopkins, Nat. Rev. Drug Discovery 5(12), 993-996 (2006).

${ }^{4}$ A. Krogh, B. Larsson, G. von Heijne, and E. L. L. Sonnhammer, J. Mol. Biol. 305(3), 567-580 (2001).

${ }^{5}$ C. W. Fong, J. Membr. Biol. 248(4), 651-669 (2015).

${ }^{6}$ S. M. Loverde, J. Phys. Chem. Lett. 5(10), 1659-1665 (2014).

${ }^{7}$ D. J. Müller, A. Engel, U. Matthey, T. Meier, P. Dimroth, and K. Suda, J. Mol. Biol. 327(5), 925-930 (2003).

${ }^{8}$ N. K. Sarangi, N. Ramesh, and A. Patnaik, J. Chem. Phys. 142(2), 024702 (2015).

${ }^{9}$ K. Nakagawa, Langmuir 19(12), 5078-5082 (2003).

${ }^{10}$ M. Kansy, F. Senner, and K. Gubernator, J. Med. Chem. 41(7), 1007-1010 (1998).

${ }^{11}$ M. Thompson, U. J. Krull, and P. J. Worsfold, Anal. Chim. Acta 117, 133-145 (1980).

${ }^{12}$ M. Thompson, U. J. Krull, and P. J. Worsfold, Anal. Chim. Acta 117, 121-132 (1980).

${ }^{13}$ M. Thompson and U. J. Krull, Anal. Chim. Acta 141, 49-56 (1982).

${ }^{14}$ D. P. Tieleman, Clin. Exp. Pharmacol. Physiol. 33(10), 893-903 (2006).

${ }^{15}$ S.-J. Marrink and H. J. C. Berendsen, J. Phys. Chem. 98(15), 4155-4168 (1994).

${ }^{16}$ F. Y. Hansen, G. H. Peters, H. Taub, and A. Miskowiec, J. Chem. Phys. 137(20), 204910 (2012).

${ }^{17}$ T. Wei, T. Huang, B. Qiao, M. Zhang, H. Ma, and L. Zhang, J. Phys. Chem. B 118(46), 13202-13209 (2014)

${ }^{18}$ J. Comer, K. Schulten, and C. Chipot, J. Chem. Theory Comput. 10(7), 2710-2718 (2014)

${ }^{19}$ S. J. Marrink and H. J. C. Berendsen, J. Phys. Chem. 100(41), 16729-16738 (1996).

${ }^{20}$ M. Orsi, W. E. Sanderson, and J. W. Essex, J. Phys. Chem. B 113(35), 12019-12029 (2009).

${ }^{21}$ S. Riahi and C. N. Rowley, J. Am. Chem. Soc. 136(43), 15111-15113 (2014).

${ }^{22}$ D. Bemporad, C. Luttmann, and J. W. Essex, Biochim. Biophys. Acta, Biomembr. 1718(1-2), 1-21 (2005). 
${ }^{23}$ Y. Wang, D. Hu, and D. Wei, J. Chem. Theory Comput. 10(4), 1717-1726 (2014).

${ }^{24}$ H. E. Alper and T. R. Stouch, J. Phys. Chem. 99(15), 5724-5731 (1995).

${ }^{25}$ M. Orsi and J. W. Essex, Soft Matter 6(16), 3797 (2010).

${ }^{26}$ L. J. Martin, R. Chao, and B. Corry, Biophys. Chem. 185, 98-107 (2014).

${ }^{27}$ R. D. Porasso, W. F. Drew Bennett, S. D. Oliveira-Costa, and J. J. López Cascales, J. Phys. Chem. B 113(29), 9988-9994 (2009).

${ }^{28}$ Y. Nademi, S. A. Iranagh, A. Yousefpour, S. Z. Mousavi, and H. Modarress, J. Chem. Sci. 126(3), 637-647 (2014).

${ }^{29}$ W. Khuntawee, P. Wolschann, T. Rungrotmongkol, J. Wong-ekkabut, and S. Hannongbua, J. Chem. Inf. Model. 55(9), 1894-1902 (2015).

${ }^{30}$ D. J. V. A. dos Santos and L. A. Eriksson, Biophys. J. 91(7), 2464-2474 (2006).

${ }^{31}$ J. J. L. Cascales, M. L. Huertas, and J. G. de la Torre, Biophys. Chem. 69(1), 1-8 (1997).

${ }^{32}$ A. Kyrychenko, I. Y. Sevriukov, Z. A. Syzova, A. S. Ladokhin, and A. O. Doroshenko, Biophys. Chem. 154(1), 8-17 (2011).

${ }^{33}$ C. Wei and A. Pohorille, J. Phys. Chem. B 118(45), 12919-12926 (2014).

${ }^{34}$ T.-X. Xiang, Z.-Q. Jiang, L. Song, and B. D. Anderson, Mol. Pharmaceutics 3(5), 589-600 (2006).

${ }^{35}$ T. M. Nolte, K. Kettler, J. A. J. Meesters, A. J. Hendriks, and D. van de Meent, Environ. Toxicol. Chem. 34(3), 488-496 (2015).

${ }^{36}$ P. A. Oroskar, C. J. Jameson, and S. Murad, Langmuir 31(3), 1074-1085 (2015).

${ }^{37}$ S. L. Fiedler and A. Violi, Biophys. J. 99(1), 144-152 (2010).

${ }^{38}$ M. Casalegno, G. Raos, and G. Sello, Phys. Chem. Chem. Phys. 17(4), 2344-2348 (2015).

${ }^{39}$ H. D. Herce and A. E. Garcia, J. Biol. Phys. 33(5-6), 345-356 (2007).

${ }^{40}$ H. D. Herce and A. E. Garcia, Proc. Natl. Acad. Sci. U. S. A. 104(52), 20805-20810 (2007).

${ }^{41}$ A. Khajeh and H. Modarress, Biophys. Chem. 187-188, 43-50 (2014).

${ }^{42}$ J. A. Söderhäll and A. Laaksonen, J. Phys. Chem. B 105(38), 9308-9315 (2001).

${ }^{43}$ A. E. Cardenas, G. S. Jas, K. Y. DeLeon, W. A. Hegefeld, K. Kuczera, and R. Elber, J. Phys. Chem. B 116(9), 2739-2750 (2012).

${ }^{44}$ A. E. Cardenas, R. Shrestha, L. J. Webb, and R. Elber, J. Phys. Chem. B 119(21), 6412-6420 (2015).

${ }^{45}$ I. Wood, M. F. Martini, and M. Pickholz, J. Mol. Struct. 1045, 124-130 (2013).

${ }^{46}$ J. L. MacCallum, W. F. D. Bennett, and D. P. Tieleman, Biophys. J. 101(1), 110-117 (2011).

${ }^{47}$ D. Bonhenry, M. Tarek, and F. Dehez, J. Chem. Theory Comput. 9(12), 5675-5684 (2013).

${ }^{48}$ J. L. MacCallum, W. F. D. Bennett, and D. P. Tieleman, Biophys. J. 94(9), 3393-3404 (2008).

${ }^{49}$ M. Fujikawa, K. Nakao, R. Shimizu, and M. Akamatsu, Bioorg. Med. Chem. 15(11), 3756-3767 (2007).

${ }^{50}$ R. Ano, Y. Kimura, M. Shima, R. Matsuno, T. Ueno, and M. Akamatsu, Bioorg. Med. Chem. 12(1), 257-264 (2004).

${ }^{51}$ F. T. T. Huque, K. Box, J. A. Platts, and J. Comer, Eur. J. Pharm. Sci. 23(3), 223-232 (2004).

${ }^{52}$ A. Avdeef, S. Bendels, L. Di, B. Faller, M. Kansy, K. Sugano, and Y. Yamauchi, J. Pharm. Sci. 96(11), 2893-2909 (2007).

${ }^{53}$ A. Avdeef, P. E. Nielsen, and O. Tsinman, Eur. J. Pharm. Sci. 22(5), 365-374 (2004).

${ }^{54}$ A. Avdeef and O. Tsinman, Eur. J. Pharm. Sci. 28(1-2), 43-50 (2006).

${ }^{55}$ B. Brooks, R. Bruccoleri, B. Olafson, D. States, S. Swaminathan, and M. Karplus, J. Comput. Chem. 4(2), 187-217 (1983).

${ }^{56}$ B. R. Brooks, C. L. Brooks, A. D. MacKerell, L. Nilsson, R. J. Petrella, B. Roux, Y. Won, G. Archontis, C. Bartels, S. Boresch, A. Caflisch, L. Caves, Q. Cui, A. R. Dinner, M. Feig, S. Fischer, J. Gao, M. Hodoscek, W. Im, K. Kuczera, T. Lazaridis, J. Ma, V. Ovchinnikov, E. Paci, R. W. Pastor, C. B. Post, J. Z. Pu, M. Schaefer, B. Tidor, R. M. Venable, H. L. Woodcock, X. Wu, W. Yang, D. M. York, and M. Karplus, J. Comput. Chem. 30(10), 1545-1614 (2009).
${ }^{57}$ S. Jo, T. Kim, and W. Im, PLoS One 2(9), e880 (2007).

${ }^{58}$ E. L. Wu, X. Cheng, S. Jo, H. Rui, K. C. Song, E. M. Dávila-Contreras, Y. Qi, J. Lee, V. Monje-Galvan, R. M. Venable, J. B. Klauda, and W. Im, J. Comput. Chem. 35(27), 1997-2004 (2014).

${ }^{59}$ S. Jo, J. B. Lim, J. B. Klauda, and W. Im, Biophys. J. 97(1), 50-58 (2009).

${ }^{60}$ S. Pronk, S. Pall, R. Schulz, P. Larsson, P. Bjelkmar, R. Apostolov, M. R. Shirts, J. C. Smith, P. M. Kasson, D. van der Spoel, B. Hess, and E. Lindahl, Bioinformatics 29(7), 845-854 (2013).

${ }^{61}$ H. I. Petrache, S. Tristram-Nagle, K. Gawrisch, D. Harries, V. A. Parsegian, and J. F. Nagle, Biophys. J. 86(3), 1574-1586 (2004).

${ }^{62}$ Y. Liu and J. F. Nagle, Phys. Rev. E 69(4), 040901(R) (2004).

${ }^{63}$ R. W. Pastor and A. D. MacKerell, J. Phys. Chem. Lett. 2(13), 1526-1532 (2011).

${ }^{64}$ K. Vanommeslaeghe, E. Hatcher, C. Acharya, S. Kundu, S. Zhong, J. Shim, E. Darian, O. Guvench, P. Lopes, I. Vorobyov, and A. D. MacKerell, J. Comput. Chem. 31, 671-690 (2009).

${ }^{65}$ W. L. Jorgensen, J. Chandrasekhar, J. D. Madura, R. W. Impey, and M. L. Klein, J. Chem. Phys. 79(2), 926 (1983).

${ }^{66}$ M. P. Allen and D. J. Tildesley, Computer Simulation of Liquids (Oxford University Press, New York, 1990).

${ }^{67}$ G. Bussi, D. Donadio, and M. Parrinello, J. Chem. Phys. 126(1), 014101 (2007).

${ }^{68}$ G. Torrie and J. Valleau, J. Comput. Phys. 23(2), 187-199 (1977)

${ }^{69}$ J. Kästner, Wiley Interdiscip. Rev.: Comput. Mol. Sci. 1(6), 932-942 (2011).

${ }^{70}$ S. Kumar, D. Bouzida, R. Swendsen, P. Kollman, and J. Rosenberg, J. Comput. Chem. 13(8), 1011-1021 (1992).

${ }^{71}$ M. Souaille and B. Roux, Comput. Phys. Commun. 135(1), 40-57 (2001).

${ }^{72}$ B. Roux, Comput. Phys. Commun. 91(1-3), 275-282 (1995).

${ }^{73}$ D. J. Bicout and A. Szabo, J. Chem. Phys. 109(6), 2325-2338 (1998).

${ }^{74}$ G. Hummer, New J. Phys. 7, 34 (2005).

${ }^{75}$ R. Kubo, Rep. Prog. Phys. 29, 255-284 (1966).

${ }^{76}$ S. Nosé, J. Chem. Phys. 81(1), 511 (1984).

${ }^{77}$ W. Hoover, Phys. Rev. A 31(3), 1695-1697 (1985).

${ }^{78}$ J. M. Diamond and Y. Katz, J. Membr. Biol. 17(2), 121-154 (1974).

${ }^{79}$ K. Schulten, J. Chem. Phys. 74(8), 4426 (1981).

${ }^{80}$ J. Ulander and A. D. J. Haymet, Biophys. J. 85(6), 3475-3484 (2003).

${ }^{81}$ K. Kuczera, J. Unruh, C. K. Johnson, and G. S. Jas, J. Phys. Chem. A 114(1), 133-142 (2010)

${ }^{82}$ A. C. Chakrabarti and D. W. Deamer, Biochim. Biophys. Acta 1111(2), 171-177 (1992).

${ }^{83}$ A. C. Chakrabarti and D. W. Deamer, J. Mol. Evol. 39(1), 1-5 (1994).

${ }^{84}$ J. S. Hub, B. L. de Groot, and D. van der Spoel, J. Chem. Theory Comput. 6(12), 3713-3720 (2010).

${ }^{85}$ A. E. Cardenas and R. Elber, J. Chem. Phys. 141(5), 054101 (2014).

${ }^{86}$ S. Viswanath, S. M. Kreuzer, A. E. Cardenas, and R. Elber, J. Chem. Phys. 139(17), 174105 (2013).

${ }^{87}$ G. Parisio, M. Stocchero, and A. Ferrarini, J. Chem. Theory Comput. 9(12), 5236-5246 (2013).

${ }^{88}$ J. P. M. Jämbeck and A. P. Lyubartsev, J. Phys. Chem. Lett. 4(11), 1781-1787 (2013).

${ }^{89}$ A. E. Cardenas and R. Elber, Mol. Phys. 111(22-23), 3565-3578 (2013).

${ }^{90}$ J. Comer, K. Schulten, and C. Chipot, J. Chem. Theory Comput. 10(2), 554-564 (2014).

${ }^{91}$ J. Henin, E. Tajkhorshid, K. Schulten, and C. Chipot, Biophys. J. 94(3), 832-839 (2008).

${ }^{92}$ W. Shinoda and S. Okazaki, J. Chem. Phys. 109(4), 1517 (1998).

${ }^{93}$ C. Neale, C. Madill, S. Rauscher, and R. Pomès, J. Chem. Theory Comput. 9(8), 3686-3703 (2013).

${ }^{94}$ D. Pal and P. Chakrabarti, Biopolymers 63(3), 195-206 (2002).

${ }^{95}$ D. J. Tobias and C. L. Brooks, J. Phys. Chem. 96(9), 3864-3870 (1992).

${ }^{96}$ H. A. L. Filipe, M. J. Moreno, T. Róg, I. Vattulainen, and L. M. S. Loura, J. Phys. Chem. B 118(13), 3572-3581 (2014) 